\title{
A HIGH-ORDER DISCONTINUOUS GALERKIN METHOD FOR THE PORO-ELASTO-ACOUSTIC PROBLEM ON POLYGONAL AND POLYHEDRAL GRIDS
}

\author{
P.F. Antonietti ${ }^{1}$, M. Botti ${ }^{2}$, I. Mazzieri ${ }^{3}$, S. Nati Poltri ${ }^{4}$ \\ July 6,2022 \\ ${ }^{1}$ MOX, Dipartimento di Matematica, Politecnico di Milano, Italy. \\ paola.antonietti@polimi.it \\ 2 MOX, Dipartimento di Matematica, Politecnico di Milano, Italy. \\ michele.botti@polimi.it \\ 3 MOX, Dipartimento di Matematica, Politecnico di Milano, Italy. \\ ilario.mazzieri@polimi.it \\ ${ }^{4}$ MOX, Dipartimento di Matematica, Politecnico di Milano, Italy. \\ simone.nati@mail.polimi.it
}

Keywords: poroelasticity; acoustics; interface conditions; discontinuous Galerkin method; convergence analysis

\begin{abstract}
The aim of this work is to introduce and analyze a finite element discontinuous Galerkin method on polygonal meshes for the numerical discretization of acoustic waves propagation through poroelastic materials. Wave propagation is modeled by the acoustics equations in the acoustic domain and the low-frequency Biot's equations in the poroelastic one. The coupling is realized by means of (physically consistent) transmission conditions, imposed on the interface between the domains, modeling different pores configurations. For the space discretization we introduce and analyze a high-order discontinuous Galerkin method on polygonal and polyhedral meshes, which is then coupled with Newmark- $\beta$ time integration schemes. A stability analysis for both the continuous and semi-discrete problem is presented and error estimates for the energy norm are derived for the semi-discrete one. A wide set of numerical results obtained on test cases with manufactured solutions are presented in order to validate the error analysis. Examples of physical interest are also presented to investigate the capability of the proposed methods in practical scenarios.
\end{abstract}

\section{Introduction}

The paper deals with the numerical analysis of the coupled poro-elasto-acoustic differential problem modeling an acoustic/sound wave impacting a poroelastic medium and consequently propagating through it. Coupled poro-elasto-acoustic problems model the combined propagation of pressure and elastic waves through a porous material. Pressure waves propagate through the saturating fluid inside pores, while acoustic ones through the porous skeleton. The theory of propagation of acoustic waves with application to poroelasticity has been developed mainly by Biot [14] in 1956, by introducing general equations and proposing different ways to treat coupling between acoustic and poro-elastic domains. Pioneering advances of Biot's theory concerned with slow compressional waves, whose study carried on the analysis on fast compressional waves, 
introduced in 1944 by Frenkel. Coupled poro-elasto-acoustic models find application in many science and engineering fields. For example, in acoustic engineering, for the study of sound propagation through acoustic panels, whose main intent is to intercept and absorb acoustic waves for noise reduction [49]; in civil engineering, for the study of passive control and vibroacoustics, where plastic foams and fibrous or granular materials are mainly used with this intent [35]; in aeronautical engineering, where air-saturated porous materials are employed [22]; in biomedical engineering, for the study of ultrasound propagation throughout bones to diagnose osteoporosis and study its evolution [32] and to model soft tissues deformation, such as the heart tissue [33], the skin [39] and the aortic tissue [34]. Poro-elasto-acoustic models find a wide strand of literature also in computational geosciences: we refer the reader to [21] for a comprehensive review.

In order to model the poroelastic domain, the concept of pores is necessary. Pores can be seen as "holes" in the material where a fluid is able to move. They can be classified into open, sealed, and imperfect pores: the first ones share a part with the outer surface of the material, the second ones are totally locked in, while the latter ones represent an itermediate state between the former two, as shown in Figure 1a below. From the modeling viewpoint, the difference between them is the way in which interface conditions are formulated, as detailed later on.

Concerning the numerical discretization of poro-elasto-acoustic models, we mention the Lagrange Multipliers method [44, 2, 29], the finite element method [13, 28] the spectral and pseudospectral element method [38, 45], the ADER scheme [25, 23], the finite difference method [36], and references therein.

To accurately simulate wave propagation in coupled poro-elasto-acoustic domains the numerical scheme should take into account the following observations: (i) in the low-frequency range the evolution problem become stiff [25], and therefore, explicit time integration schemes might become computationally too demanding due to the strict stability constraint; (ii) the diffusive slow compressional waves are localized near the interfaces, and therefore, mesh refinements are needed to capture the phenomenon; (iii) an accurate geometrical description of the arbitrary complex interfaces is crucial; (iv) a proper representation of the hydraulic contact at the interfaces is also mandatory to correctly capture the physics of the problem.

By taking into consideration the aforementioned difficulties, the aim of this paper is to propose and analyze a high-order discontinuous Galerkin method on polygonal and polyhedral grids (PolyDG) for the space discretization of a coupled poroelasto-acoustic problem, by extending the theory carried out in [4], where a coupled system of elasto-acoustic equations is analyzed. We point out that the geometric flexibility due to mild regularity requirements on the underlying computational mesh together with the arbitrary-order accuracy featured by the proposed PolyDG method are crucial within this context as they ensure at the same time a high-level of flexibility in the representation of the geometry and an intrinsic high-level of precision and scalability that are mandatory to correctly represent the solution fields. Moreover, in the proposed semi-discrete formulation, the coupling between the acoustic and the poroelastic domains is introduced by considering (physically consistent) interface conditions, naturally incorporated in the scheme.

For early results in the field of $\mathrm{dG}$ methods we refer, for example, to [11, 7, 20, 18, 24, 17] for second-order elliptic problems problems, to [16] for parabolic differential equations, to [6] for flows in fractured porous media, to [3] for fluid structure interaction problems, cf. also [19] for a comprehensive monograph. In the framework of $\mathrm{dG}$ methods for hyperbolic problems we mention $[43,30]$ for scalar wave equation on simplex grids, while more recent dG discretizations on polytopic meshes can be found in [8] for elastodynamics problems, in [9] for non-linear sound waves and in $[4,5]$ for coupled elasto-acoustic problems. To the best of our knowledge, the present approach is proposed and analyzed here for the first time in the context of multiphysics poroelasto-acoustic problems, and it provides a flexible and accurate scheme that can be employed in real applications. 
Figure 1: (1a) Pores classification in a poroelastic domain: sealed (1), open (2) and imperfect (3) pores. (1b) Simplified graphic representation of the domain $\Omega=\Omega_{p} \cup \Omega_{a}$ for $d=2$.

(a) Pores classification in a poroelastic domain.

(b) $\Omega=\Omega_{p} \cup \Omega_{a}$.
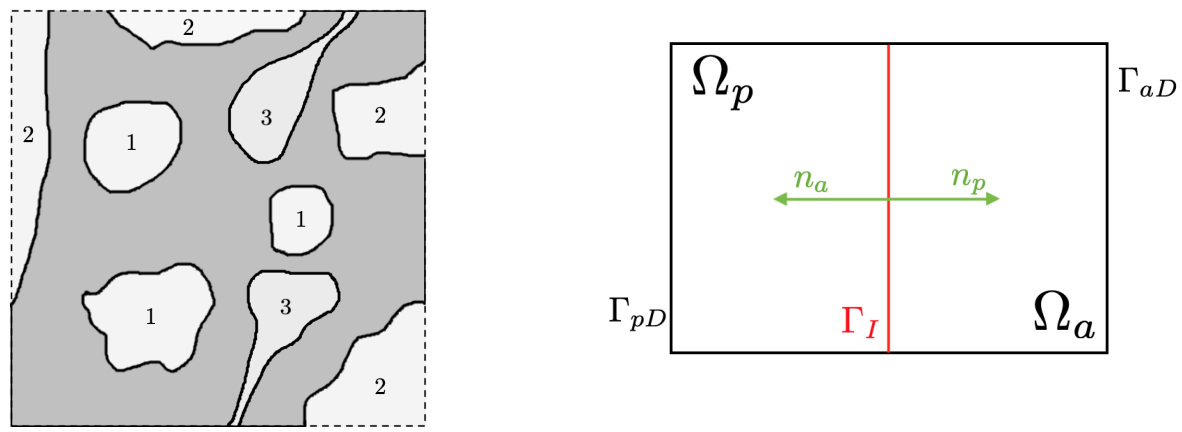

The remaining part of the paper is structured as follows: in Section 2 we introduce the mathematical model, present the weak formulation of the problem, and prove suitable stability estimates. In Section 3 we introduce the PolyDG approximation and prove its stability. Section 4 is devoted to the analysis of the semi-discrete problem and the proof of $h p$-version a-priori error estimates. The time integration schemes are introduced in Section 5. In Section 6 we present some two-dimensional numerical experiments to validate the theoretical results and show the performances of the proposed method in examples of physical interest. Finally, in Section 7 we draw some conclusions. The existence and uniqueness for the strong formulation of the problem and additional technical results are established in Appendix A.

\section{The physical model and governing equations}

Let $\Omega \subset \mathbb{R}^{d}, d=2,3$, be an open, convex polygonal/polyhedral domain decomposed as the union of two disjoint, polygonal/polyhedral subdomains: $\Omega=\Omega_{p} \cup \Omega_{a}$, representing the poroelastic and the acoustic domains, respectively, cf. Figure 1b. The two subdomains share part of their boundary, resulting in the interface $\Gamma_{I}=\partial \Omega_{p} \cap \partial \Omega_{a}$. The boundary of $\Omega$ is denoted by $\partial \Omega$, and we set $\partial \Omega_{p}=\Gamma_{p D} \cup \Gamma_{I}$ and $\partial \Omega_{a}=\Gamma_{a D} \cup \Gamma_{I}$, with $\Gamma_{p D} \cap \Gamma_{I}=\emptyset$ and $\Gamma_{a D} \cap \Gamma_{I}=\emptyset$. Surface measures of $\partial \Omega, \partial \Omega_{p}, \partial \Omega_{a}$ and $\Gamma_{I}$ are assumed to be strictly positive. The outer unit normal vectors to $\partial \Omega_{p}$ and $\partial \Omega_{a}$ are denoted by $\boldsymbol{n}_{p}$ and $\boldsymbol{n}_{a}$, respectively, so that $\boldsymbol{n}_{p}=-\boldsymbol{n}_{a}$ on $\Gamma_{I}$. In the following, for $X \subseteq \Omega$, the notation $\boldsymbol{L}^{2}(X)$ is adopted in place of $\left[L^{2}(X)\right]^{d}$, with $d \in\{2,3\}$. The scalar product in $L^{2}(X)$ is denoted by $(\cdot, \cdot)_{X}$, with associated norm $\|\cdot\|_{X}$. Similarly, $\boldsymbol{H}^{\ell}(X)$ is defined as $\left[H^{\ell}(X)\right]^{d}$, with $\ell \geq 0$, equipped with the norm $\|\cdot\|_{\ell, X}$, assuming conventionally that $\boldsymbol{H}^{0}(X) \equiv \boldsymbol{L}^{2}(X)$. In addition we will use $\boldsymbol{H}(\operatorname{div}, X)$ to denote the space of $\boldsymbol{L}^{2}(X)$ functions with square integrable divergence. In order to take into account essential boundary conditions, we also introduce the zero-trace subspaces, defined as

$$
\begin{aligned}
H_{0}^{1}\left(\Omega_{a}\right) & =\left\{\psi \in H^{1}\left(\Omega_{a}\right) \mid \psi_{\mid \Gamma_{a D}}=0\right\}, \\
\boldsymbol{H}_{0}^{1}\left(\Omega_{p}\right) & =\left\{\boldsymbol{v} \in \boldsymbol{H}^{1}\left(\Omega_{p}\right) \mid \boldsymbol{v}_{\mid \Gamma_{p D}}=\mathbf{0}\right\}, \\
\boldsymbol{H}_{0}\left(\operatorname{div}, \Omega_{p}\right) & =\left\{\boldsymbol{z} \in \boldsymbol{H}\left(\operatorname{div}, \Omega_{p}\right) \mid\left(\boldsymbol{z} \cdot \boldsymbol{n}_{p}\right)_{\mid \Gamma_{p D}}=0\right\} .
\end{aligned}
$$

Given $k \in \mathbb{N}$ and a Hilbert space $\mathbb{H}$, the usual notation $C^{k}([0, T] ; \mathbb{H})$ is adopted for the space of $\mathbb{H}$-valued functions, $k$-times continuously differentiable in $[0, T]$. The notation $x \lesssim y$ stands 
for $x \leq C y$, with $C>0$, independent of the discretization parameters, but possibly dependent on physical coefficients and the final time $T$.

\subsection{The poro-elasto-acoustic problem}

To model wave propagation in a poro-elastic domain $\Omega_{p}$ we consider the two-displacement formulation of [37], written in the solid and filtration displacements, denoted by $\boldsymbol{u}$ and $\boldsymbol{w}$, respectively. For a final observation time $T>0$, we consider the low-frequency Biot's equations:

$$
\begin{cases}\rho \ddot{\boldsymbol{u}}+\rho_{f} \ddot{\boldsymbol{w}}-\nabla \cdot \boldsymbol{\sigma}=\boldsymbol{f}_{p}, & \text { in } \Omega_{p} \times(0, T], \\ \rho_{f} \ddot{\boldsymbol{u}}+\rho_{w} \ddot{\boldsymbol{w}}+\frac{\eta}{k} \dot{\boldsymbol{w}}+\nabla p=\boldsymbol{g}_{p}, & \text { in } \Omega_{p} \times(0, T]\end{cases}
$$

Here, the average density $\rho$ is given by $\rho=\phi \rho_{f}+(1-\phi) \rho_{s}$, where $\rho_{s}>0$ is the solid density, $\rho_{f}>0$ is the saturating fluid density, $\rho_{w}$ is defined as $\rho_{w}=\frac{a}{\phi} \rho_{f}$, being $\phi$ the porosity satisfying $0<\phi_{0} \leq \phi \leq \phi_{1}<1$, and being $a>1$ the tortuosity measuring the deviation of the fluid paths from straight streamlines, cf. [46]. In (1), $\eta>0$ represents the dynamic viscosity of the fluid and $k>0$ is the absolute permeability.

Remark 2.1. As observed in [23], the second equation in (1) is valid under a constraint on frequencies, i.e. the spectrum of the waves has to lie in the low-frequency range. In what follows, we only consider frequencies lower than $f_{c}=\eta \phi /\left(2 \pi a k \rho_{f}\right)$.

In $\Omega_{p}$, we assume the following constitutive laws for the stress $\boldsymbol{\sigma}$ and pressure $p$ :

$$
\boldsymbol{\sigma}(\boldsymbol{u}, p)=\mathbb{C}: \boldsymbol{\epsilon}(\boldsymbol{u})-\beta p \boldsymbol{I}, \quad p(\boldsymbol{u}, \boldsymbol{w})=-m(\beta \nabla \cdot \boldsymbol{u}+\nabla \cdot \boldsymbol{w}),
$$

where the strain tensor $\boldsymbol{\epsilon}(\cdot)$ is defined as $\boldsymbol{\epsilon}(\boldsymbol{u})=\frac{1}{2}\left(\nabla \boldsymbol{u}+\nabla \boldsymbol{u}^{T}\right)$, and $\mathbb{C}$ is the fourth-order, symmetric and uniformly elliptic elasticity tensor defined by

$$
\mathbb{C}: \boldsymbol{\tau}=2 \mu \boldsymbol{\tau}+\lambda \operatorname{tr}(\boldsymbol{\tau}), \quad \text { for all } \boldsymbol{\tau} \in \mathbb{R}^{\mathrm{d} \times \mathrm{d}},
$$

with $\operatorname{tr}(\boldsymbol{\tau})=\sum_{\mathrm{i}=1}^{\mathrm{d}} \boldsymbol{\tau}_{\mathrm{ii}}$. Here, $\lambda \geq 0$ and $\mu \geq \mu_{0}>0$ are the Lamé coefficients of the elastic skeleton. In 2, the Biot-Willis coefficient $\beta$ and Biot modulus $m$ are such that $\phi<\beta \leq 1$ and $m \geq m_{0}>0$. It can be shown that the dilatation coefficients of the saturated matrix corresponds to $\lambda_{f}=\lambda+\beta^{2} m$. By plugging the constitutive laws (2) into (1), we obtain the two-displacement formulation

$$
\left\{\begin{array}{l}
\rho \ddot{\boldsymbol{u}}+\rho_{f} \ddot{\boldsymbol{w}}-\nabla \cdot(\mathbb{C}: \boldsymbol{\epsilon}(\boldsymbol{u}))-\beta^{2} m \nabla(\nabla \cdot \boldsymbol{u})-\beta m \nabla(\nabla \cdot \boldsymbol{w})=\boldsymbol{f}_{p}, \\
\rho_{f} \ddot{\boldsymbol{u}}+\rho_{w} \ddot{\boldsymbol{w}}+\frac{\eta}{k} \dot{\boldsymbol{w}}-\beta m \nabla(\nabla \cdot \boldsymbol{u})-m \nabla(\nabla \cdot \boldsymbol{w})=\boldsymbol{g}_{p} .
\end{array}\right.
$$

Remark 2.2. We point out that the $(\boldsymbol{u}, \boldsymbol{w})$ formulation (3) is not the unique possible choice. For example, one could write the equations considering the velocity of the solid skeleton $\dot{\boldsymbol{u}}$ and the filtration velocity $\dot{\boldsymbol{w}}$ as unknowns, cf. [23], or consider a velocity-pressure $(\boldsymbol{u}, p)$ formulation, as in $[2,12,15,41]$. Here, the two-displacement formulation turns out to be convenient in view of the coupling conditions stated below.

In the fluid domain $\Omega_{a}$, we consider an acoustic wave with constant velocity $c>0$ and mass density $\rho_{a}>0$. For a given source term $f_{a}$, the acoustic potential $\varphi$ satisfies

$$
c^{-2} \ddot{\varphi}-\rho_{a}^{-1} \nabla \cdot\left(\rho_{a} \nabla \varphi\right)=f_{a}, \quad \text { in } \Omega_{a} \times(0, T] .
$$

Finally, we discuss the transmission conditions on $\Gamma_{I}$. The poro-elasto-acoustic coupling is realized through interface conditions, cf. [31], expressing the continuity of normal stresses and 
conservation of mass. The continuity of the pressure is prescribed by writing the acoustic potential in terms of a pressure. Thus, on $\Gamma_{I}$ we impose

$$
\begin{aligned}
-\boldsymbol{\sigma} \boldsymbol{n}_{p} & =\rho_{a} \dot{\varphi} \boldsymbol{n}_{p}, \\
(\dot{\boldsymbol{u}}+\dot{\boldsymbol{w}}) \cdot \boldsymbol{n}_{p} & =-\nabla \varphi \cdot \boldsymbol{n}_{p}, \\
\tau[p] & =(1-\tau) \dot{\boldsymbol{w}} \cdot \boldsymbol{n}_{p},
\end{aligned}
$$

where $[\cdot]$ denotes the jump operator at the interface $\Gamma_{I}$, i.e. $[p]=p(\boldsymbol{u}, \boldsymbol{w})-p_{a}(\varphi)$ with $p_{a}(\varphi)=$ $\rho_{a} \dot{\varphi}$, and $0 \leq \tau \leq 1$ is the hydraulic permeability at the interface and models both open, sealed, and imperfect pores, cf. 1a. The stress tensor $\boldsymbol{\sigma}$ and the pressure $p(\boldsymbol{u}, \boldsymbol{w})$ obey the constitutive equations (2). If $\tau=1$ (open pores), equation (7) reduces to the continuity of pressure at the interface, that is $p(\boldsymbol{u}, \boldsymbol{w})=\rho_{a} \dot{\varphi}$. If $\tau=0$ (sealed pores), (7) simplifies to $\dot{\boldsymbol{w}} \cdot \boldsymbol{n}_{p}=0$, that implies that (6) imposes a continuity only on the solid velocity, namely $\dot{\boldsymbol{u}} \cdot \boldsymbol{n}_{p}=-\nabla \varphi \cdot \boldsymbol{n}_{p}$. If $\tau \in(0,1)$ (imperfect pores) then an intermediate state between open and sealed pores occurs.

Supplementing the constitutive equations with suitable boundary conditions (here supposed for simplicity to be of homogeneous Dirichlet type), the poro-elasto-acoustic problem reads as: for any $t \in(0, T]$, find $(\boldsymbol{u}, \boldsymbol{w}, \varphi): \Omega_{p} \times \Omega_{p} \times \Omega_{a} \rightarrow \mathbb{R}$ such that:

$$
\begin{aligned}
\rho \ddot{\boldsymbol{u}}+\rho_{f} \ddot{\boldsymbol{w}}-\nabla \cdot(\mathbb{C}: \boldsymbol{\epsilon}(\boldsymbol{u}))-\beta m \nabla(\beta \nabla \cdot \boldsymbol{u}+\nabla \cdot \boldsymbol{w}) & =\boldsymbol{f}_{p}, & & \text { in } \Omega_{p}, \\
\rho_{f} \ddot{\boldsymbol{u}}+\rho_{w} \ddot{\boldsymbol{w}}+\frac{\eta}{k} \dot{\boldsymbol{w}}-m \nabla(\beta \nabla \cdot \boldsymbol{u}+\nabla \cdot \boldsymbol{w}) & =\boldsymbol{g}_{p}, & & \text { in } \Omega_{p} \\
\rho_{a} c^{-2} \ddot{\varphi}-\nabla \cdot\left(\rho_{a} \nabla \varphi\right) & =\rho_{a} f_{a} & & \text { in } \Omega_{a}, \\
-(\mathbb{C}: \boldsymbol{\epsilon}(\boldsymbol{u})+\beta m(\beta \nabla \cdot \boldsymbol{u}+\nabla \cdot \boldsymbol{w}) \boldsymbol{I}) \boldsymbol{n}_{p} & =\rho_{a} \dot{\varphi} \boldsymbol{n}_{p}, & & \text { on } \Gamma_{I}, \\
(\dot{\boldsymbol{u}}+\dot{\boldsymbol{w}}) \cdot \boldsymbol{n}_{p} & =-\nabla \varphi \cdot \boldsymbol{n}_{p}, & & \text { on } \Gamma_{I}, \\
-m(\beta \nabla \cdot \boldsymbol{u}+\nabla \cdot \boldsymbol{w})-\tau^{-1}(1-\tau) \dot{\boldsymbol{w}} \cdot \boldsymbol{n}_{p} & =\rho_{a} \dot{\varphi}, & & \text { on } \Gamma_{I},
\end{aligned}
$$

together with initial conditions $\boldsymbol{u}(\cdot, 0)=\boldsymbol{u}_{0}, \boldsymbol{w}(\cdot, 0)=\boldsymbol{w}_{0}, \dot{\boldsymbol{u}}(\cdot, 0)=\boldsymbol{u}_{1}, \dot{\boldsymbol{w}}(\cdot, 0)=\boldsymbol{w}_{1}$, in $\Omega_{p}$ and $\varphi(\cdot, 0)=\varphi_{0}, \dot{\varphi}(\cdot, 0)=\varphi_{1}$ in $\Omega_{a}$. Notice that the acoustic equation has been multiplied by $\rho_{a}$. The existence and uniqueness of a strong solution to (8) is proved in Appendix A by employing the semigroup theory.

\subsection{Weak formulation and stability estimates}

In order to derive a unified analysis for $0 \leq \tau \leq 1$, we introduce the space

$$
\boldsymbol{W}_{\tau}= \begin{cases}\boldsymbol{H}_{0}\left(\operatorname{div}, \Omega_{p}\right), & \text { if } \tau=1, \\ \left\{\boldsymbol{z} \in \boldsymbol{H}_{0}\left(\operatorname{div}, \Omega_{p}\right) \mid \zeta(\tau)^{\frac{1}{2}}\left(\boldsymbol{z} \cdot \boldsymbol{n}_{p}\right)_{\mid \Gamma_{I}} \in L^{2}\left(\Gamma_{I}\right)\right\}, & \text { if } \tau \in(0,1), \\ \left\{\boldsymbol{z} \in \boldsymbol{H}_{0}\left(\operatorname{div}, \Omega_{p}\right) \mid\left(\boldsymbol{z} \cdot \boldsymbol{n}_{p}\right)_{\mid \Gamma_{I}}=0\right\}, & \text { if } \tau=0,\end{cases}
$$

equipped with the norm $\|\cdot\|_{\boldsymbol{W}_{\tau}}$ defined, for all $\boldsymbol{z} \in \boldsymbol{W}_{\tau}$, as

$$
\|\boldsymbol{z}\|_{\boldsymbol{W}_{\tau}}=\|\boldsymbol{z}\|_{\Omega_{p}}+\|\nabla \cdot \boldsymbol{z}\|_{\Omega_{p}}+\left\|\zeta(\tau)^{\frac{1}{2}} \boldsymbol{z} \cdot \boldsymbol{n}_{p}\right\|_{\Gamma_{I}}, \quad \text { with } \zeta(\tau)= \begin{cases}\frac{1-\tau}{\tau} & \text { for } \tau \in(0,1] \\ 0 & \text { for } \tau=0\end{cases}
$$

We also define the Hilbert space $\mathbb{H}=\boldsymbol{H}_{0}^{1}\left(\Omega_{p}\right) \times \boldsymbol{W}_{\tau} \times H_{0}^{1}\left(\Omega_{a}\right)$ and $\Omega_{*}=\Omega_{p} \times \Omega_{p} \times \Omega_{a}$. The weak form of $(8)$ reads as: for any $t \in(0, T]$, find $(\boldsymbol{u}, \boldsymbol{w}, \varphi)(t) \in \mathbb{H}$ s.t.

$$
\begin{aligned}
\mathcal{M}((\ddot{\boldsymbol{u}}, \ddot{\boldsymbol{w}}, \ddot{\varphi}),(\boldsymbol{v}, \boldsymbol{z}, \psi))+\mathcal{A}((\boldsymbol{u}, \boldsymbol{w}, \varphi),(\boldsymbol{v}, \boldsymbol{z}, \psi))+\mathcal{B}(\dot{\boldsymbol{w}}, \boldsymbol{z}) & \\
& +\mathcal{C}^{p}(\dot{\varphi}, \boldsymbol{v}+\boldsymbol{z})+\mathcal{C}^{a}(\dot{\boldsymbol{u}}+\dot{\boldsymbol{w}}, \psi)=\left(\left(\boldsymbol{f}_{p}, \boldsymbol{g}_{p}, \rho_{a} f_{a}\right),(\boldsymbol{v}, \boldsymbol{z}, \psi)\right)_{\Omega_{*}}
\end{aligned}
$$


for all $(\boldsymbol{v}, \boldsymbol{z}, \psi) \in \mathbb{H}$, where for any $\mathfrak{U}=(\boldsymbol{u}, \boldsymbol{w}, \varphi), \mathfrak{V}=(\boldsymbol{v}, \boldsymbol{z}, \psi) \in \mathbb{H}$ we have set

$$
\begin{aligned}
\mathcal{M}(\mathfrak{U}, \mathfrak{V}) & =\left(\rho \boldsymbol{u}+\rho_{f} \boldsymbol{w}, \boldsymbol{v}\right)_{\Omega_{p}}+\left(\rho_{f} \boldsymbol{u}+\rho_{w} \boldsymbol{w}, \boldsymbol{z}\right)_{\Omega_{p}}+\left(\rho_{a} c^{-2} \varphi, \psi\right)_{\Omega_{a}}, \\
\mathcal{A}(\mathfrak{U}, \mathfrak{V}) & =(\mathbb{C}: \boldsymbol{\epsilon}(\boldsymbol{u}), \boldsymbol{\epsilon}(\boldsymbol{v}))_{\Omega_{p}}+(m \nabla \cdot(\beta \boldsymbol{u}+\boldsymbol{w}), \nabla \cdot(\beta \boldsymbol{v}+\boldsymbol{z}))_{\Omega_{p}}+\left(\rho_{a} \nabla \varphi, \nabla \psi\right)_{\Omega_{a}}, \\
\mathcal{B}(\boldsymbol{w}, \boldsymbol{z}) & =\left(\eta k^{-1} \boldsymbol{w}, \boldsymbol{z}\right)_{\Omega_{p}}+\left(\zeta(\tau) \boldsymbol{w} \cdot \boldsymbol{n}_{p}, \boldsymbol{z} \cdot \boldsymbol{n}_{p}\right)_{\Gamma_{I}}, \\
\mathcal{C}^{p}(\varphi, \boldsymbol{z}) & =\left\langle\rho_{a} \varphi, \boldsymbol{z} \cdot \boldsymbol{n}_{p}\right\rangle_{\Gamma_{I}}=-\mathcal{C}^{a}(\boldsymbol{z}, \varphi),
\end{aligned}
$$

with $\zeta(\tau)$ defined in (10). Notice that, if $\tau=0$, the terms $\mathcal{C}^{p}(\dot{\varphi}, z)$ and $\mathcal{C}^{a}(\dot{\boldsymbol{w}}, \psi)$ in (11) are null thanks to the definition of $\boldsymbol{W}_{\tau}$ which strongly enforces condition (7).

Before presenting a stability estimate for the solution of problem (11) we define, for all $\mathfrak{U}=(\boldsymbol{u}, \boldsymbol{w}, \varphi) \in C^{1}\left([0, T] ; \boldsymbol{L}^{2}\left(\Omega_{\star}\right)\right) \cap C^{0}([0, T] ; \mathbb{H})$, the energy norm

$$
\|\mathfrak{U}\|_{\mathbb{E}}^{2}=\max _{t \in[0, T]}\|\mathfrak{U}(t)\|_{\mathcal{E}}^{2}=\max _{t \in(0, T]}(\mathcal{M}(\dot{\mathfrak{U}}, \dot{\mathfrak{U}})(t)+\mathcal{A}(\mathfrak{U}, \mathfrak{U})(t)+\mathcal{B}(\boldsymbol{w}, \boldsymbol{w})(t)) .
$$

As a result of the next Lemma, $\|\cdot\|_{\mathbb{E}}$ is a norm on $C^{1}\left([0, T] ; \boldsymbol{L}^{2}\left(\Omega_{\star}\right)\right) \cap C^{0}([0, T] ; \mathbb{H})$.

Lemma 1. The bilinear forms $\mathcal{M}, \mathcal{A}$, and $\mathcal{B}$ defined in (12) are such that

$$
\begin{aligned}
\mathcal{M}(\mathfrak{U}, \mathfrak{V}) & \lesssim\|\mathfrak{U}\|_{\Omega_{*}}\|\mathfrak{V}\|_{\Omega_{*}}, \\
\mathcal{M}(\mathfrak{U}, \mathfrak{U}) & \gtrsim\|\mathfrak{U}\|_{\Omega_{*}}^{2}, \\
\mathcal{A}(\mathfrak{U}, \mathfrak{V})+\mathcal{B}(\boldsymbol{w}, \boldsymbol{z}) & \lesssim\|\boldsymbol{u}\|_{1, \Omega_{p}}\|\boldsymbol{v}\|_{1, \Omega_{p}}+\|\boldsymbol{w}\|_{\boldsymbol{W}_{\tau}}\|\boldsymbol{z}\|_{\boldsymbol{W}_{\tau}}+\|\varphi\|_{1, \Omega_{a}}\|\psi\|_{1, \Omega_{a}}, \\
\mathcal{A}(\mathfrak{U}, \mathfrak{U})+\mathcal{B}(\boldsymbol{w}, \boldsymbol{w}) & \gtrsim\|\boldsymbol{u}\|_{1, \Omega_{p}}^{2}+\|\boldsymbol{w}\|_{\boldsymbol{W}_{\tau}}^{2}+\|\varphi\|_{1, \Omega_{a}}^{2},
\end{aligned}
$$

for any $\mathfrak{U}=(\boldsymbol{u}, \boldsymbol{w}, \varphi), \mathfrak{V}=(\boldsymbol{v}, \boldsymbol{z}, \psi) \in \mathbb{H}$.

Proof. Inequalities (14) and (16) are readily inferred by applying the Cauchy-Schwarz and triangle inequalities, while (15) is obtained by noting that $\rho \rho_{w}-\rho_{f}^{2}>0$ and $\rho_{a} c^{-2}>0$. The last inequality (17) represents the $\mathbb{H}$-coercivity of $\mathcal{A}(\cdot, \cdot)+\mathcal{B}(\cdot, \cdot)$. To prove this property we apply Poincaré's and Korn's inequalities in $H_{0}^{1}\left(\Omega_{a}\right)$ and $\boldsymbol{H}_{0}^{1}\left(\Omega_{p}\right)$, respectively, to infer $\|\boldsymbol{u}\|_{1, \Omega_{p}}^{2}+$ $\|\varphi\|_{1, \Omega_{a}}^{2} \lesssim \mathcal{A}(\mathfrak{U}, \mathfrak{U})$. Then, using the triangle inequality and recalling definition (10) of the $\boldsymbol{W}_{\tau}$-norm we get

$$
\|\boldsymbol{w}\|_{\boldsymbol{W}_{\tau}}^{2} \lesssim\|\nabla \cdot(\beta \boldsymbol{u}+\boldsymbol{w})\|_{\Omega_{p}}^{2}+\|\beta \nabla \cdot \boldsymbol{u}\|_{\Omega_{p}}^{2}+\mathcal{B}(\boldsymbol{w}, \boldsymbol{w}) \lesssim \mathcal{A}(\mathfrak{U}, \mathfrak{U})+\mathcal{B}(\boldsymbol{w}, \boldsymbol{w})
$$

and the conclusion follows.

Theorem 1 (Stability of the continuous weak formulation). Assume that the problem data satisfy $\left(\boldsymbol{f}_{p}, \boldsymbol{g}_{p}, \rho_{a} f_{a}\right) \in L^{2}\left((0, T) ; \boldsymbol{L}^{2}\left(\Omega_{*}\right)\right), \mathfrak{U}(0)=\left(\boldsymbol{u}_{0}, \boldsymbol{w}_{0}, \varphi_{0}\right) \in \mathbb{H}$, and $\dot{\mathfrak{U}}(0)=\left(\boldsymbol{u}_{1}, \boldsymbol{w}_{1}, \varphi_{1}\right) \in$ $\boldsymbol{L}^{2}\left(\Omega_{*}\right)$. For any $t \in(0, T]$, let $\mathfrak{U}(t)=(\boldsymbol{u}, \boldsymbol{w}, \varphi)(t) \in \mathbb{H}$ be the solution of (11). Then, it holds

$$
\|\mathfrak{U}(t)\|_{\mathcal{E}}^{2} \lesssim\|\mathfrak{U}(0)\|_{\mathcal{E}}^{2}+\int_{0}^{T}\left\|\left(\boldsymbol{f}_{p}, \boldsymbol{g}_{p}, \rho_{a} f_{a}\right)(s)\right\|_{\Omega_{*}}^{2} d s,
$$

with the hidden constant depending on the observation time $t \leq T$ and on the material properties, but independent of $\tau$.

Proof. Taking $\dot{\mathfrak{U}}=(\dot{\boldsymbol{u}}, \dot{\boldsymbol{w}}, \dot{\varphi})$ as test functions in (11), using $\mathcal{C}^{a}(\dot{\boldsymbol{u}}+\dot{\boldsymbol{w}}, \dot{\varphi})+\mathcal{C}^{p}(\dot{\varphi}, \dot{\boldsymbol{u}}+\dot{\boldsymbol{w}})=0$, and integrating in time between 0 and $t \leq T$, it is inferred that

$$
\mathcal{M}(\dot{\mathfrak{U}}, \dot{\mathfrak{U}})(t)+\mathcal{A}(\mathfrak{U}, \mathfrak{U})(t)+\int_{0}^{t} 2 \mathcal{B}(\dot{\boldsymbol{w}}, \dot{\boldsymbol{w}}) d s=\mathcal{M}(\dot{\mathfrak{U}}, \dot{\mathfrak{U}})(0)+\mathcal{A}(\mathfrak{U}, \mathfrak{U})(0)+\int_{0}^{t} 2(\mathfrak{F}, \dot{\mathfrak{U}})_{\Omega_{*}} d s
$$

where we have adopted the abridged notation $\mathfrak{F}=\left(\boldsymbol{f}_{p}, \boldsymbol{g}_{p}, \rho_{a} f_{a}\right)$. Hence, applying the CauchySchwarz and Young inequalities to bound the third term in the right-hand side, using that 
$\mathcal{B}(\boldsymbol{w}, \boldsymbol{w})(t) \leq \mathcal{B}(\boldsymbol{w}, \boldsymbol{w})(0)+\int_{0}^{t} \mathcal{B}(\dot{\boldsymbol{w}}, \dot{\boldsymbol{w}})(s) d s$, and recalling definition (13) of the energy norm, for all $t \in(0, T]$ one has

$$
\|\mathfrak{U}(t)\|_{\mathcal{E}}^{2} \lesssim\|\mathfrak{U}(0)\|_{\mathcal{E}}^{2}+\int_{0}^{t}\|\mathfrak{F}(s)\|_{\Omega_{*}}^{2} d s+\int_{0}^{t}\|\dot{U}(s)\|_{\Omega_{*}}^{2} d s .
$$

Finally, owing to (14), we obtain $\|\dot{\mathfrak{U}}\|_{\Omega_{*}}^{2} \lesssim\|\mathfrak{U}\|_{\mathcal{E}}^{2}$, so that the thesis follows by applying the Gronwall's Lemma [42].

\section{The semi-discrete formulation and its stability analysis}

We introduce a polytopic mesh $\mathcal{T}_{h}$ made of general polygons (in $2 \mathrm{~d}$ ) or polyhedra (in $3 \mathrm{~d}$ ) and write $\mathcal{T}_{h}$ as $\mathcal{T}_{h}=\mathcal{T}_{h}^{p} \cup \mathcal{T}_{h}^{a}$, where $\mathcal{T}_{h}^{\#}=\left\{\kappa \in \mathcal{T}_{h}: \bar{\kappa} \subseteq \bar{\Omega}_{\#}\right\}$, with \# $=\{p, a\}$. Implicit in this decomposition there is the assumption that the meshes $\mathcal{T}_{h}^{a}$ and $\mathcal{T}_{h}^{p}$ are aligned with $\Omega_{a}$ and $\Omega_{p}$, respectively. Polynomial degrees $p_{p, \kappa} \geq 1$ and $p_{a, \kappa} \geq 1$ are associated with each element of $\mathcal{T}_{h}^{p}$ and $\mathcal{T}_{h}^{a}$, respectively. The discrete spaces are introduced as follows: $\boldsymbol{V}_{h}^{p}=\left[\mathcal{P}_{p_{p}}\left(\mathcal{T}_{h}^{p}\right)\right]^{d}$ and $V_{h}^{a}=\mathcal{P}_{p_{a}}\left(\mathcal{T}_{h}^{a}\right)$, where $\mathcal{P}_{r}\left(\mathcal{T}_{h}^{\#}\right)$ is the space of piecewise polynomials in $\Omega_{\#}$ of degree less than or equal to $r$ in any $\kappa \in \mathcal{T}_{h}^{\#}$ with $\#=\{p, a\}$.

In the following, we assume that $\mathbb{C}, \rho_{a}$ and $m$ are element-wise constant and we define $\overline{\mathbb{C}}_{\kappa}=\left(\left|\mathbb{C}^{1 / 2}\right|_{2}^{2}\right)_{\mid \kappa}, \bar{m}_{\kappa}=(m)_{\mid \kappa}$ for all $\kappa \in \mathcal{T}_{h}^{p}$ and $\bar{\rho}_{a, \kappa}=\rho_{a \mid \kappa}$ for all $\kappa \in \mathcal{T}_{h}^{a}$. The symbol $|\cdot|_{2}$ stands for the $\ell^{2}$-norm on $\mathbb{R}^{n \times n}$, with $n=3$ if $d=2$ and $n=6$ if $d=3$. In order to deal with polygonal and polyhedral elements, we define an interface as the intersection of the $(d-1)$-dimensional faces of any two neighboring elements of $\mathcal{T}_{h}$. If $d=2$, an interface/face is a line segment and the set of all interfaces/faces is denoted by $\mathcal{F}_{h}$. When $d=3$, an interface can be a general polygon that we assume could be further decomposed into a set of planar triangles collected in the set $\mathcal{F}_{h}$. We decompose $\mathcal{F}_{h}$ as $\mathcal{F}_{h}=\mathcal{F}_{h}^{I} \cup \mathcal{F}_{h}^{p} \cup \mathcal{F}_{h}^{a}$, where $\mathcal{F}_{h}^{I}=\left\{F \in \mathcal{F}_{h}: F \subset \partial \kappa^{p} \cap \partial \kappa^{a}, \kappa^{p} \in \mathcal{T}_{h}^{p}, \kappa^{a} \in \mathcal{T}_{h}^{a}\right\}$, and $\mathcal{F}_{h}^{p}$ and $\mathcal{F}_{h}^{a}$ denote all the faces of $\mathcal{T}_{h}^{p}$ and $\mathcal{T}_{h}^{a}$, respectively, not laying on $\Gamma_{I}$. Finally, the faces of $\mathcal{T}_{h}^{p}$ and $\mathcal{T}_{h}^{a}$ can be further written as the union of internal $(i)$ and boundary $(b)$ faces, respectively, i.e.: $\mathcal{F}_{h}^{p}=\mathcal{F}_{h}^{p, i} \cup \mathcal{F}_{h}^{p, b}$ and $\mathcal{F}_{h}^{a}=\mathcal{F}_{h}^{a, i} \cup \mathcal{F}_{h}^{a, b}$.

Following [19], we next introduce the main assumption on $\mathcal{T}_{h}$.

Definition 3.1. A mesh $\mathcal{T}_{h}$ is said to be polytopic-regular if for any $\kappa \in \mathcal{T}_{h}$, there exists a set of non-overlapping $d$-dimensional simplices contained in $\kappa$, denoted by $\left\{S_{\kappa}^{F}\right\}_{F \subset \partial \kappa}$, such that for any face $F \subset \partial \kappa$, the following condition holds:

$$
h_{\kappa} \lesssim d\left|S_{\kappa}^{F}\right||F|^{-1}
$$

Assumption 3.1. The sequence of meshes $\left\{\mathcal{T}_{h}\right\}_{h}$ is assumed to be uniformly polytopic regular in the sense of Definition 3.1.

As pointed out in [19], this assumption does not impose any restriction on either the number of faces per element nor their measure relative to the diameter of the element they belong to. Under Assumption 3.1, the following trace-inverse inequality holds:

$$
\|v\|_{L^{2}(\partial \kappa)} \lesssim p h_{\kappa}^{-1 / 2}\|v\|_{L^{2}(\kappa)} \quad \forall \kappa \in \mathcal{T}_{h} \forall v \in \mathcal{P}_{p}(\kappa) .
$$

In order to avoid technicalities, we also make the following assumption.

Assumption 3.2. For any pair of neighboring elements $\kappa^{ \pm} \in \mathcal{T}_{h}$. The following $h p$-local bounded variation property holds: $h_{\kappa^{+}} \lesssim h_{\kappa^{-}} \lesssim h_{\kappa^{+}}, p_{\kappa^{+}} \lesssim p_{\kappa^{-}} \lesssim p_{\kappa^{+}}$. 
Finally, following [10], for sufficiently piecewise smooth scalar-, vector- and tensor-valued fields $\psi, \boldsymbol{v}$ and $\boldsymbol{\tau}$, respectively, we define the averages and jumps on each interior face $F \in$ $\mathcal{F}_{h}^{p, i} \cup \mathcal{F}_{h}^{a, i} \cup \mathcal{F}_{h}^{I}$ shared by the elements $\kappa^{ \pm} \in \mathcal{T}_{h}^{p}$ as follows:

$$
\begin{aligned}
& \llbracket \psi \rrbracket=\psi^{+} \boldsymbol{n}^{+}+\psi^{-} \boldsymbol{n}^{-}, \quad \llbracket \boldsymbol{v} \rrbracket=\boldsymbol{v}^{+} \otimes \boldsymbol{n}^{+}+\boldsymbol{v}^{-} \otimes \boldsymbol{n}^{-}, \quad \llbracket \boldsymbol{v} \rrbracket_{\boldsymbol{n}}=\boldsymbol{v}^{+} \cdot \boldsymbol{n}^{+}+\boldsymbol{v}^{-} \cdot \boldsymbol{n}^{-}, \\
& \{\psi\}=\frac{\psi^{+}+\psi^{-}}{2}, \quad\{\boldsymbol{v}\}=\frac{\boldsymbol{v}^{+}+\boldsymbol{v}^{-}}{2}, \quad\{\boldsymbol{\tau}\}=\frac{\boldsymbol{\tau}^{+}+\boldsymbol{\tau}^{-}}{2},
\end{aligned}
$$

where $\otimes$ is the tensor product in $\mathbb{R}^{3},{ }^{ \pm}$denotes the trace on $F$ taken within $\kappa^{ \pm}$, and $\boldsymbol{n}^{ \pm}$ is the outer normal vector to $\partial \kappa^{ \pm}$. Accordingly, on boundary faces $F \in \mathcal{F}_{h}^{p, b} \cup \mathcal{F}_{h}^{a, b}$, we set $\llbracket \psi \rrbracket=\psi \boldsymbol{n},\{\psi\}=\psi, \llbracket \boldsymbol{v} \rrbracket=\boldsymbol{v} \otimes \boldsymbol{n}, \llbracket \boldsymbol{v} \rrbracket_{\boldsymbol{n}}=\boldsymbol{v} \cdot \boldsymbol{n},\{\boldsymbol{v}\}=\boldsymbol{v},\{\boldsymbol{\tau}\}=\boldsymbol{\tau}$.

\subsection{Semi-discrete PolyDG formulation}

We are now ready to introduce the semi-discrete formulation: for $t \in(0, T]$, find $\left(\boldsymbol{u}_{h}, \boldsymbol{w}_{h}, \varphi_{h}\right)(t) \in$ $\boldsymbol{V}_{h}^{p} \times \boldsymbol{V}_{h}^{p} \times V_{h}^{a}$, s.t.

$$
\begin{aligned}
\mathcal{M}\left(\left(\ddot{\boldsymbol{u}}_{h}, \ddot{\boldsymbol{w}}_{h}, \ddot{\varphi}_{h}\right),\left(\boldsymbol{v}_{h}, \boldsymbol{z}_{h}, \psi_{h}\right)\right)+\mathcal{A}_{h}\left(\left(\boldsymbol{u}_{h}, \boldsymbol{w}_{h}, \varphi_{h}\right),\left(\boldsymbol{v}_{h}, \boldsymbol{z}_{h}, \psi_{h}\right)\right)+\mathcal{B}\left(\dot{\boldsymbol{w}}_{h}, \boldsymbol{z}_{h}\right) \\
+\mathcal{C}_{h}^{p}\left(\dot{\varphi}_{h}, \boldsymbol{v}_{h}+\boldsymbol{z}_{h}\right)+\mathcal{C}_{h}^{a}\left(\dot{\boldsymbol{u}}_{h}+\dot{\boldsymbol{w}}_{h}, \psi_{h}\right)=\left(\left(\boldsymbol{f}_{p}, \boldsymbol{g}_{p}, \rho_{a} f_{a}\right),\left(\boldsymbol{v}_{h}, \boldsymbol{\xi}_{h}, \psi_{h}\right)\right)_{\Omega_{*}}
\end{aligned}
$$

for all $\left(\boldsymbol{v}_{h}, \boldsymbol{\xi}_{h}, \psi_{h}\right) \in \boldsymbol{V}_{h}^{p} \times \boldsymbol{V}_{h}^{p} \times V_{h}^{a}$. As initial conditions we take the $L^{2}$-orthogonal projections onto $\left(\boldsymbol{V}_{h}^{p} \times \boldsymbol{V}_{h}^{p} \times V_{h}^{a}\right)^{2}$ of the initial data $\left(\boldsymbol{u}_{0}, \boldsymbol{w}_{0}, \varphi_{0}, \boldsymbol{u}_{1}, \boldsymbol{w}_{1}, \varphi_{1}\right)$. We define $\nabla_{h}$ and $\nabla_{h}$. to be the broken gradient and divergence operators, respectively, set $\boldsymbol{\epsilon}_{h}(\boldsymbol{v})=\frac{\nabla_{h} \boldsymbol{v}+\nabla_{h} \boldsymbol{v}^{T}}{2}, \boldsymbol{\sigma}_{h}(\boldsymbol{v})=$ $\mathbb{C}: \boldsymbol{\epsilon}_{h}(\boldsymbol{v})$, and use the short-hand notation $(\cdot, \cdot)_{\Omega_{\#}}=\sum_{\kappa \in \mathcal{T}_{h}^{\#}} \int_{\kappa} \cdot$ and $\langle\cdot, \cdot\rangle_{\mathcal{F}_{h}^{\#}}=\sum_{F \in \mathcal{F}_{h}^{\#}} \int_{F} \cdot$ for $\#=\{a, p\}$. Then, for all $\boldsymbol{u}, \boldsymbol{v}, \boldsymbol{w}, \boldsymbol{z} \in \boldsymbol{V}_{h}^{p}$ and $\varphi, \psi \in V_{h}^{a}$, the bilinear forms appearing in the above formulation are given by

$$
\begin{aligned}
\mathcal{A}_{h}((\boldsymbol{u}, \boldsymbol{v}, \varphi),(\boldsymbol{v}, \boldsymbol{z}, \psi)) & =\mathcal{A}_{h}^{e}(\boldsymbol{u}, \boldsymbol{v})+\mathcal{A}_{h}^{p}(\beta \boldsymbol{u}+\boldsymbol{w}, \beta \boldsymbol{v}+\boldsymbol{z})+\mathcal{A}_{h}^{a}(\varphi, \psi), \\
\mathcal{C}_{h}^{p}(\varphi, \boldsymbol{v}) & =\left\langle\rho_{a} \varphi, \boldsymbol{v} \cdot \boldsymbol{n}_{p}\right\rangle_{\mathcal{F}_{h}^{I}}=-\mathcal{C}_{h}^{a}(\boldsymbol{v}, \varphi)
\end{aligned}
$$

with

$$
\begin{gathered}
\mathcal{A}_{h}^{e}(\boldsymbol{u}, \boldsymbol{v})=\left(\boldsymbol{\sigma}_{h}(\boldsymbol{u}), \boldsymbol{\epsilon}_{h}(\boldsymbol{v})\right)_{\Omega_{p}}-\left\langle\left\{\boldsymbol{\sigma}_{h}(\boldsymbol{u})\right\}, \llbracket \boldsymbol{v} \rrbracket\right\rangle_{\mathcal{F}_{h}^{p}} \\
-\left\langle\llbracket \boldsymbol{u} \rrbracket,\left\{\left\{\boldsymbol{\sigma}_{h}(\boldsymbol{v})\right\}\right\rangle_{\mathcal{F}_{h}^{p}}+\langle\alpha \llbracket \boldsymbol{u} \rrbracket, \llbracket \boldsymbol{v} \rrbracket\rangle_{\mathcal{F}_{h}^{p}},\right. \\
\mathcal{A}_{h}^{p}(\boldsymbol{w}, \boldsymbol{z})=\left(m \nabla_{h} \cdot \boldsymbol{w}, \nabla_{h} \cdot \boldsymbol{z}\right)_{\Omega_{p}}-\left\langle\left\{m\left(\nabla_{h} \cdot \boldsymbol{w}\right)\right\}, \llbracket \boldsymbol{z} \rrbracket_{\boldsymbol{n}}\right\rangle_{\mathcal{F}_{h}^{\star}} \\
-\left\langle\llbracket \boldsymbol{w} \rrbracket_{\boldsymbol{n}},\left\{m\left(\nabla_{h} \cdot \boldsymbol{z}\right)\right\}\right\rangle_{\mathcal{F}_{h}^{\star}}+\left\langle\gamma \llbracket \boldsymbol{w} \rrbracket_{\boldsymbol{n}}, \llbracket \boldsymbol{z} \rrbracket_{\boldsymbol{n}}\right\rangle_{\mathcal{F}_{h}^{\star}}, \\
\mathcal{A}_{h}^{a}(\varphi, \psi)=\left(\rho_{a} \nabla_{h} \varphi, \nabla_{h} \psi\right)_{\Omega_{a}}-\left\langle\left\{\left\{\rho_{a} \nabla_{h} \varphi \rrbracket, \llbracket \psi \rrbracket\right\rangle_{\mathcal{F}_{h}^{a}}^{a}\right.\right. \\
-\left\langle\llbracket \varphi \rrbracket,\left\{\rho_{a} \nabla_{h} \psi\right\}\right\rangle_{\mathcal{F}_{h}^{a}}+\langle\chi \llbracket \varphi \rrbracket, \llbracket \psi \rrbracket\rangle_{\mathcal{F}_{h}^{a}},
\end{gathered}
$$

and $\mathcal{F}_{h}^{\star}=\mathcal{F}_{h}^{p}$ in the case $\tau \in(0,1]$, while $\mathcal{F}_{h}^{\star}=\mathcal{F}_{h}^{p} \cup \mathcal{F}_{h}^{I}$ in the case $\tau=0$. The stabilization functions $\alpha \in L^{\infty}\left(\mathcal{F}_{h}^{p}\right), \gamma \in L^{\infty}\left(\mathcal{F}_{h}^{p}\right)$ and $\chi \in L^{\infty}\left(\mathcal{F}_{h}^{a}\right)$, are defined s.t.

$$
\begin{gathered}
\left.\alpha\right|_{F}=\left\{\begin{array}{lll}
c_{1} \max _{\kappa \in\left\{\kappa^{+}, \kappa^{-}\right\}}\left(\overline{\mathbb{C}}_{\kappa} p_{p, \kappa}^{2} h_{\kappa}^{-1}\right) & \forall F \in \mathcal{F}_{h}^{p, i}, & F \subseteq \partial \kappa^{+} \cap \partial \kappa^{-}, \\
\overline{\mathbb{C}}_{\kappa} p_{p, \kappa}^{2} h_{\kappa}^{-1} & \forall F \in \mathcal{F}_{h}^{p, b}, & F \subseteq \partial \kappa,
\end{array}\right. \\
\left.\gamma\right|_{F}= \begin{cases}c_{2} \max _{\kappa \in\left\{\kappa^{+}, \kappa^{-}\right\}}\left(\bar{m}_{\kappa} p_{p, \kappa}^{2} h_{\kappa}^{-1}\right) & \forall F \in \mathcal{F}_{h}^{p, i}, \quad F \subseteq \partial \kappa^{+} \cap \partial \kappa^{-}, \\
\bar{m}_{\kappa} p_{p, \kappa}^{2} h_{\kappa}^{-1} & \forall F \in \mathcal{F}_{h}^{p, b} \cup \mathcal{F}_{h}^{I}, F \subseteq \partial \kappa,\end{cases}
\end{gathered}
$$




$$
\left.\chi\right|_{F}=\left\{\begin{array}{lll}
c_{3} \max _{\kappa \in\left\{\kappa^{+}, \kappa^{-}\right\}}\left(\bar{\rho}_{a, \kappa} p_{a, \kappa}^{2} h_{\kappa}^{-1}\right) & \forall F \in \mathcal{F}_{h}^{a, i}, & F \subseteq \partial \kappa^{+} \cap \partial \kappa^{-}, \\
\bar{\rho}_{a, \kappa} p_{a, \kappa}^{2} h_{\kappa}^{-1} & \forall F \in \mathcal{F}_{h}^{a, b}, \quad F \subseteq \partial \kappa,
\end{array}\right.
$$

with $c_{1}, c_{2}, c_{3}>0$ positive constants, to be properly chosen. The definition of the penalty functions (23)-(25) is based on [19, Lemma 35]. With this choice, the bilinear forms in (23) are symmetric and coercive, cf. Lemma A.2. Alternative stabilization functions can be defined in the spirit of [1]. The analysis of the latter is however beyond the scope of this work. See also [26] for the elliptic case.

By fixing a basis for $\boldsymbol{V}_{h}^{p}$ and $V_{h}^{a}$ and denoting by $(U, W, \Phi)$ the vector of the expansion coefficients in the chosen basis of the unknowns $\boldsymbol{u}_{h}, \boldsymbol{w}_{h}$ and $\varphi_{h}$, respectively, the semi-discrete formulation (20) can be written equivalently as:

$$
\begin{aligned}
{\left[\begin{array}{ccc}
\rho \boldsymbol{M}^{p} & \rho_{f} \boldsymbol{M}^{p} & 0 \\
\rho_{f} \boldsymbol{M}^{p} & \rho_{w} \boldsymbol{M}^{p} & 0 \\
0 & 0 & \rho_{a} c^{-2} \boldsymbol{M}^{a}
\end{array}\right]\left[\begin{array}{c}
\ddot{U} \\
\ddot{W} \\
\ddot{\Phi}
\end{array}\right]+\left[\begin{array}{ccc}
0 & 0 & \boldsymbol{C}^{p} \\
0 & \boldsymbol{B} & \boldsymbol{C}^{p} \\
\boldsymbol{C}^{a} & \boldsymbol{C}^{a} & 0
\end{array}\right]\left[\begin{array}{c}
\dot{U} \\
\dot{W} \\
\dot{\Phi}
\end{array}\right] } \\
+\left[\begin{array}{ccc}
\boldsymbol{A}^{e}+\beta^{2} \boldsymbol{A}^{p} & \beta \boldsymbol{A}^{p} & 0 \\
\beta \boldsymbol{A}^{p} & \boldsymbol{A}^{p} & 0 \\
0 & 0 & \boldsymbol{A}^{a}
\end{array}\right]\left[\begin{array}{c}
U \\
W \\
\Phi
\end{array}\right]=\left[\begin{array}{c}
\boldsymbol{F}^{p} \\
\boldsymbol{G}^{p} \\
\boldsymbol{F}^{a}
\end{array}\right]
\end{aligned}
$$

with initial conditions $U(0)=U_{0}, W(0)=W_{0}, \Phi(0)=\Phi_{0}, \dot{U}(0)=U_{1}, \dot{W}(0)=W_{1}, \dot{\Phi}(0)=\Phi_{1}$. We remark that $\boldsymbol{F}^{p}, \boldsymbol{G}^{p}$ and $\boldsymbol{F}^{a}$ are the vector representations of the linear functionals $\left(\boldsymbol{f}_{p}, \boldsymbol{v}_{h}\right)_{\Omega_{p}}$, $\left(\boldsymbol{g}_{p}, \boldsymbol{\xi}_{h}\right)_{\Omega_{p}}$ and $\left(\rho_{a} f_{a}, \psi_{h}\right)_{\Omega_{a}}$, respectively.

\subsection{Stability analysis}

To carry out the stability analysis of the semi-discrete problem, we introduce the energy norm

$$
\begin{aligned}
\|(\boldsymbol{v}, \boldsymbol{z}, \psi)(t)\|_{\mathrm{E}}^{2}=\mathcal{M}((\dot{\boldsymbol{v}}, \dot{\boldsymbol{z}}, \dot{\psi}),(\dot{\boldsymbol{v}}, \dot{\boldsymbol{z}}, \dot{\psi}))(t) & +\mathcal{B}(\boldsymbol{z}, \boldsymbol{z})(t) \\
& +\|\boldsymbol{v}(t)\|_{\mathrm{dG}, \mathrm{e}}^{2}+|(\beta \boldsymbol{v}+\boldsymbol{z})(t)|_{\mathrm{dG}, \mathrm{p}}^{2}+\|\psi(t)\|_{\mathrm{dG}, \mathrm{a}}^{2}
\end{aligned}
$$

for all $(\boldsymbol{v}, \boldsymbol{z}, \psi) \in C^{1}\left([0, T] ; \boldsymbol{V}_{h}^{p} \times \boldsymbol{V}_{h}^{p} \times V_{h}^{a}\right)$, where

$$
\begin{aligned}
\|\boldsymbol{v}\|_{\mathrm{dG}, \mathrm{e}}^{2} & =\left\|\mathbb{C}^{1 / 2}: \boldsymbol{\epsilon}_{h}(\boldsymbol{v})\right\|_{\Omega_{p}}^{2}+\left\|\alpha^{1 / 2} \llbracket \boldsymbol{v} \rrbracket\right\|_{\mathcal{F}_{h}^{p}}^{2} & & \forall \boldsymbol{v} \in \boldsymbol{V}_{h}^{p}, \\
|\boldsymbol{z}|_{\mathrm{dG}, \mathrm{p}}^{2} & =\left\|m^{1 / 2} \nabla_{h} \cdot \boldsymbol{z}\right\|_{\Omega_{p}}^{2}+\left\|\gamma^{1 / 2} \llbracket \boldsymbol{z} \rrbracket_{\boldsymbol{n}}\right\|_{\mathcal{F}_{h}^{\star}}^{2} & & \forall \boldsymbol{z} \in \boldsymbol{V}_{h}^{p}, \\
\|\psi\|_{\mathrm{dG}, \mathrm{a}}^{2} & =\left\|\rho_{a}{ }^{1 / 2} \nabla_{h} \psi\right\|_{\Omega_{a}}^{2}+\left\|\chi^{1 / 2} \llbracket \psi \rrbracket\right\|_{\mathcal{F}_{h}^{a}}^{2} & & \forall \psi \in V_{h}^{a} .
\end{aligned}
$$

Remark 3.1. The notation $|\cdot|_{\mathrm{dG}, \mathrm{p}}$ is used instead of $\|\cdot\|_{\mathrm{dG}, \mathrm{p}}$ in order to highlight that $|\cdot|_{\mathrm{dG}, \mathrm{p}}$ : $\boldsymbol{V}_{h}^{p} \rightarrow \mathbb{R}^{+}$is a seminorm. However, by proceeding as in the proof of (17), we can show that $\|\boldsymbol{v}\|_{\mathrm{dG}, \mathrm{e}}^{2}+|\beta \boldsymbol{v}+\boldsymbol{z}|_{\mathrm{dG}, \mathrm{p}}^{2}+\mathcal{B}(\boldsymbol{z}, \boldsymbol{z})$ is a norm on $\boldsymbol{V}_{h}^{p} \times \boldsymbol{V}_{h}^{p}$.

Remark 3.2. Notice that the norm defined in (27) represents the mechanical energy of the poroelasto-acoustic system. We observe that in the case of null external forces, i.e., $\boldsymbol{f}_{p}=\boldsymbol{g}_{p}=\mathbf{0}$ and $f_{a}=0$, estimate (28) reduces to $\left\|\left(\boldsymbol{u}_{h}, \boldsymbol{w}_{h}, \varphi_{h}\right)(t)\right\|_{\mathrm{E}} \lesssim\left\|\left(\boldsymbol{u}_{h}, \boldsymbol{w}_{h}, \varphi_{h}\right)(0)\right\|_{\mathrm{E}}$ for any $t>0$, namely the $\mathrm{dG}$ formulation (20) is dissipative.

The main stability result is stated in the following theorem. 
Theorem 2 (Stability of the semi-discrete formulation). Let Assumptions 3.1 and 3.2 be satisfied. For sufficiently large penalty parameters $c_{1}, c_{2}$ and $c_{3}$ in (23), (24) and (25), respectively, let $\left(\boldsymbol{u}_{h}, \boldsymbol{w}_{h}, \varphi_{h}\right)(t) \in \boldsymbol{V}_{h}^{p} \times \boldsymbol{V}_{h}^{p} \times V_{h}^{a}$ be the solution of (20) for any $t \in(0, T]$. Then, it holds

$$
\left\|\left(\boldsymbol{u}_{h}, \boldsymbol{w}_{h}, \varphi_{h}\right)(t)\right\|_{\mathrm{E}} \lesssim\left\|\left(\boldsymbol{u}_{h}, \boldsymbol{w}_{h}, \varphi_{h}\right)(0)\right\|_{\mathrm{E}}+\int_{0}^{t}\left\|\left(\boldsymbol{f}_{p}, \boldsymbol{g}_{p}, \rho_{a} f_{a}\right)(s)\right\|_{\Omega_{*}}^{2} d s,
$$

where the hidden constant depends on time $t$ and on the material properties, but is independent of $\tau$.

Proof. By taking $\left(\boldsymbol{v}_{h}, \boldsymbol{z}_{h}, \psi_{h}\right)=\left(\dot{\boldsymbol{u}}_{h}, \dot{\boldsymbol{w}}_{h}, \dot{\varphi}_{h}\right) \in \boldsymbol{V}_{h}^{p} \times \boldsymbol{V}_{h}^{p} \times V_{h}^{a}$ in (20) and using the skewsymmetry of the coupling bilinear forms (22), we obtain

$$
\begin{aligned}
\frac{1}{2} \frac{d}{d t}\left[\mathcal{M}\left(\left(\dot{\boldsymbol{u}}_{h}, \dot{\boldsymbol{w}}_{h}, \dot{\varphi}_{h}\right),\left(\dot{\boldsymbol{u}}_{h}, \dot{\boldsymbol{w}}_{h}, \dot{\varphi}_{h}\right)\right)+\mathcal{A}_{h}\left(\left(\boldsymbol{u}_{h}, \boldsymbol{v}_{h}, \varphi_{h}\right),\left(\boldsymbol{u}_{h}, \boldsymbol{v}_{h}, \varphi_{h}\right)\right)\right] \\
+\mathcal{B}\left(\dot{\boldsymbol{w}}_{h}, \dot{\boldsymbol{w}}_{h}\right)=\left(\left(\boldsymbol{f}_{p}, \boldsymbol{g}_{p}, \rho_{a} f_{a}\right),\left(\dot{\boldsymbol{u}}_{h}, \dot{\boldsymbol{z}}_{h}, \dot{\varphi}_{h}\right)\right)_{\Omega_{*}} .
\end{aligned}
$$

Thus, integrating in time between 0 and $t \leq T$, recalling definition (21) of $\mathcal{A}_{h}$, using the coercivity results of Lemma A.2, and reasoning as in the proof of Theorem 1, one can easily obtain the thesis.

\section{Error analysis for the semi-discrete formulation}

In this section we prove an a-priori error estimate for the semi-discrete problem (20). We first observe that by setting, for any time $t \in(0, T], \boldsymbol{e}^{u}(t)=\left(\boldsymbol{u}-\boldsymbol{u}_{h}\right)(t), \boldsymbol{e}^{w}(t)=\left(\boldsymbol{w}-\boldsymbol{w}_{h}\right)(t)$, and $e^{\varphi}(t)=\left(\varphi-\varphi_{h}\right)(t)$ and by using the strong consistency of the semi-discrete formulation (20), the error equation reads as follows

$$
\begin{aligned}
\mathcal{M}\left(\left(\ddot{\boldsymbol{e}}^{u}, \ddot{\boldsymbol{e}}^{w}, \ddot{e}^{\varphi}\right),(\boldsymbol{v}, \boldsymbol{z}, \psi)\right)+\mathcal{A}_{h}\left(\left(\boldsymbol{e}^{u}, \boldsymbol{e}^{w}, e^{\varphi}\right),(\boldsymbol{v}, \boldsymbol{z}, \psi)\right)+\mathcal{B}\left(\dot{\boldsymbol{e}}^{w}, \boldsymbol{z}\right) \\
+\mathcal{C}_{h}^{p}\left(\dot{e}^{\varphi}, \boldsymbol{v}+\boldsymbol{z}\right)+\mathcal{C}_{h}^{a}\left(\dot{\boldsymbol{e}}^{u}+\dot{\boldsymbol{e}}^{w}, \psi\right)=0
\end{aligned}
$$

for any $(\boldsymbol{v}, \boldsymbol{z}, \psi) \in \boldsymbol{V}_{h}^{p} \times \boldsymbol{V}_{h}^{p} \times V_{h}^{a}$. Next, we introduce the following definition and a further mesh assumption; $\mathrm{cf}[20,19]$.

Definition 4.1. A covering $\mathcal{T}_{\S}=\{\mathcal{K}\}$ of the polytopic mesh $\mathcal{T}_{h}$ is a set of regular shaped $d$-dimensional simplices $\mathcal{K}, d=2,3$, s.t. $\forall \kappa \in \mathcal{T}_{h}, \exists \mathcal{K} \in \mathcal{T}_{\S}$ s.t. $\kappa \subseteq \mathcal{K}$.

Assumption 4.1. Any mesh $\mathcal{T}_{h}$ admits a covering $\mathcal{T}_{\S}$ in the sense of (4.1) such that i) $\max _{\kappa \in \mathcal{T}_{h}} \operatorname{card}\left\{\kappa^{\prime} \in \mathcal{T}_{h}: \kappa^{\prime} \cap \mathcal{K} \neq \emptyset, \mathcal{K} \in \mathcal{T}_{\S}\right.$ s.t. $\left.\kappa \subset \mathcal{K}\right\} \lesssim 1$ and ii) $h_{\mathcal{K}} \lesssim h_{\kappa}$ for each pair $\kappa \in \mathcal{T}_{h}, \mathcal{K} \in \mathcal{T}_{\S}$ with $\kappa \subset \mathcal{K}$.

We also introduce the norm

$$
\|(\boldsymbol{v}, \boldsymbol{z}, \psi)\|_{\mathrm{E}}^{2}=\mathcal{M}((\dot{\boldsymbol{v}}, \dot{\boldsymbol{z}}, \dot{\psi}),(\dot{\boldsymbol{v}}, \dot{\boldsymbol{z}}, \dot{\psi}))+\|(\boldsymbol{v}, \boldsymbol{z}, \psi)\|_{\mathrm{dG}}^{2}+\mathcal{B}(\boldsymbol{z}, \boldsymbol{z}),
$$

where the seminorm $\|(\boldsymbol{v}, \boldsymbol{z}, \psi)\|_{\mathrm{dG}}^{2}=\|\boldsymbol{v}\|_{\mathrm{dG}, \mathrm{e}}^{2}+\|\boldsymbol{z}\|_{\mathrm{dG}, \mathrm{p}}^{2}+\|\psi\|_{\mathrm{dG}, \mathrm{a}}^{2}$ is defined by

$$
\begin{array}{ll}
\|\boldsymbol{v}\|_{\mathrm{dG}, \mathrm{e}}^{2}=\|\boldsymbol{v}\|_{\mathrm{dG}, \mathrm{e}}^{2}+\left\|\alpha^{-1 / 2}\left\{\mathbb{C}: \boldsymbol{\epsilon}_{h}(\boldsymbol{v})\right\}\right\|_{\mathcal{F}_{h}^{p}}^{2} & \forall \boldsymbol{v} \in \boldsymbol{H}^{2}\left(\mathcal{T}_{h}^{p}\right), \\
\|\boldsymbol{z}\|_{\mathrm{dG}, \mathrm{p}}^{2}=|\boldsymbol{z}|_{\mathrm{dG}, \mathrm{p}}^{2}+\left\|\gamma^{-1 / 2}\left\{\left(m \nabla_{h} \cdot \boldsymbol{z}\right)\right\}\right\|_{\mathcal{F}_{h}^{\star}}^{2} & \forall \boldsymbol{z} \in \boldsymbol{H}^{2}\left(\mathcal{T}_{h}^{p}\right), \\
\|\psi\|\left\|_{\mathrm{dG}, \mathrm{a}}^{2}=\right\| \psi\left\|_{\mathrm{dG}, \mathrm{a}}^{2}+\right\| \chi^{-1 / 2}\left\{\rho_{a} \nabla_{h} \psi\right\} \|_{\mathcal{F}_{h}^{a}}^{2} & \forall \psi \in H^{2}\left(\mathcal{T}_{h}^{a}\right) .
\end{array}
$$


For an open bounded polytopic domain $\Sigma \subset \mathbb{R}^{d}$ and a generic polytopic mesh $\mathcal{T}_{h}$ over $\Sigma$ satisfying Assumption 4.1, as in [20], we can introduce the Stein extension operator $\tilde{\mathcal{E}}: H^{m}(\kappa) \rightarrow H^{m}\left(\mathbb{R}^{d}\right)$ [47], for any $\kappa \in \mathcal{T}_{h}$ and $m \in \mathbb{N}_{0}$, such that $\left.\tilde{\mathcal{E}} v\right|_{\kappa}=v$ and $\|\tilde{\mathcal{E}} v\|_{m, \mathbb{R}^{d}} \lesssim\|v\|_{m, \kappa}$. The corresponding vector-valued version mapping $\boldsymbol{H}^{m}(\kappa)$ onto $\boldsymbol{H}^{m}\left(\mathbb{R}^{d}\right)$ acts component-wise and is denoted in the same way. In what follows, for any $\kappa \in \mathcal{T}_{h}$, we will denote by $\mathcal{K}_{\kappa}$ the simplex belonging to $\mathcal{T}_{\S}$ such that $\kappa \subset \mathcal{K}_{\kappa}$.

In order to handle the case of small interface permeability, i.e. $0<\tau<<1$, we make an additional assumption on the discretization. This requirement is consistent with the observations of [23], showing that there is a threshold value $\bar{\tau}$ such that the results for $\tau \leq \bar{\tau}$ cannot be distinguished from the sealed pores case $\tau=0$.

Assumption 4.2. In the case $\tau \in(0,1)$, for each $F \in \mathcal{F}_{h}^{I}$ and $\kappa \in \mathcal{T}_{h}^{p}$ such that $F \subset \partial \kappa \cap \Gamma_{I}$, it holds $\zeta(\tau)=\tau^{-1}(1-\tau) \lesssim h_{\kappa}^{-1} p_{p, \kappa}^{2}$, with the hidden constant independent of $\tau$. We point out that this assumption is used only for the following theoretical results but it is not needed in practice, cf. Section 6 .

The next Lemma provides the interpolation bounds that are instrumental for the derivation of the a-priori error estimate.

Lemma 2. For any $(\boldsymbol{v}, \boldsymbol{z}, \psi) \in \boldsymbol{H}^{m}\left(\mathcal{T}_{h}^{p}\right) \times \boldsymbol{H}^{\ell}\left(\mathcal{T}_{h}^{p}\right) \times H^{n}\left(\mathcal{T}_{h}^{a}\right)$, with $m, \ell, n \geq 2$, there exists $\left(\boldsymbol{v}_{I}, \boldsymbol{z}_{I}, \psi_{I}\right) \in \boldsymbol{V}_{h}^{p} \times \boldsymbol{V}_{h}^{p} \times V_{h}^{a}$ such that

$$
\begin{aligned}
\left\|\boldsymbol{v}-\boldsymbol{v}_{I}\right\|_{\mathrm{dG}, \mathrm{e}}^{2} & \lesssim \sum_{\kappa \in \mathcal{T}_{h}^{p}} \frac{h_{\kappa}^{2\left(s_{\kappa}-1\right)}}{p_{p, \kappa}^{2 m-3}}\|\widetilde{\mathcal{E}} \boldsymbol{v}\|_{m, \mathcal{K}_{\kappa}}^{2}, \\
\left\|\boldsymbol{z}-\boldsymbol{z}_{I}\right\|_{\mathrm{dG}, \mathrm{p}}^{2} & \lesssim \sum_{\kappa \in \mathcal{T}_{h}^{p}} \frac{h_{\kappa}^{2\left(r_{\kappa}-1\right)}}{p_{p, \kappa}^{2 \ell-3}}\|\widetilde{\mathcal{E}} \boldsymbol{z}\|_{\ell, \mathcal{K}_{\kappa}}^{2}, \\
\left\|\psi-\psi_{I}\right\|_{\mathrm{dG}, \mathrm{a}}^{2} & \lesssim \sum_{\kappa \in \mathcal{T}_{h}^{a}} \frac{h_{\kappa}^{2\left(q_{\kappa}-1\right)}}{p_{a, \kappa}^{2 n-3}}\|\widetilde{\mathcal{E}} \psi\|_{n, \mathcal{K}_{\kappa}}^{2},
\end{aligned}
$$

where $s_{\kappa}=\min \left(m, p_{p, \kappa}+1\right), r_{\kappa}=\min \left(\ell, p_{p, \kappa}+1\right)$ and $q_{\kappa}=\min \left(n, p_{a, \kappa}+1\right)$. Moreover, if $(\boldsymbol{u}, \boldsymbol{w}, \varphi) \in C^{1}\left([0, T] ; \boldsymbol{H}^{m}\left(\mathcal{T}_{h}^{p}\right) \times \boldsymbol{H}^{\ell}\left(\mathcal{T}_{h}^{p}\right) \times H^{n}\left(\mathcal{T}_{h}^{a}\right)\right)$, with m, $\ell, n \geq 2$, there exists $\left(\boldsymbol{u}_{I}, \boldsymbol{w}_{I}, \varphi_{I}\right) \in$ $C^{1}\left([0, T] ; \boldsymbol{V}_{h}^{p} \times \boldsymbol{V}_{h}^{p} \times V_{h}^{a}\right)$ s.t.:

$$
\begin{aligned}
\left\|\left(\boldsymbol{u}-\boldsymbol{u}_{I}, \boldsymbol{w}-\boldsymbol{w}_{I}, \varphi-\varphi_{I}\right)\right\|_{\mathrm{E}}^{2} \lesssim & \sum_{\kappa \in \mathcal{T}_{h}^{p}} \frac{h_{\kappa}^{2\left(s_{\kappa}-1\right)}}{p_{p, \kappa}^{2 m-3}}\left(\|\widetilde{\mathcal{E}} \dot{\boldsymbol{u}}\|_{m, \mathcal{K}_{\kappa}}^{2}+\|\widetilde{\mathcal{E}} \boldsymbol{u}\|_{m, \mathcal{K}_{\kappa}}^{2}\right) \\
& +\sum_{\kappa \in \mathcal{T}_{h}^{p}} \frac{h_{\kappa}^{2\left(r_{\kappa}-1\right)}}{p_{p, \kappa}^{2 \ell-3}}\left(\|\widetilde{\mathcal{E}} \dot{\boldsymbol{w}}\|_{\ell, \mathcal{K}_{\kappa}}^{2}+\|\widetilde{\mathcal{E}} \boldsymbol{w}\|_{\ell, \mathcal{K}_{\kappa}}^{2}\right) \\
& +\sum_{\kappa \in \mathcal{T}_{h}^{a}} \frac{h_{\kappa}^{2\left(q_{\kappa}-1\right)}}{p_{a, \kappa}^{2 n-3}}\left(\|\widetilde{\mathcal{E}} \dot{\varphi}\|_{n, \mathcal{K}_{\kappa}}^{2}+\|\widetilde{\mathcal{E}} \varphi\|_{n, \mathcal{K}_{\kappa}}^{2}\right) .
\end{aligned}
$$

Proof. The first part of the proof readily follows by reasoning as in [4, Lemma 5.1] and observing that $\|\cdot \cdot\|_{\mathrm{dG}, \mathrm{p}} \lesssim\|\cdot \cdot\|_{\mathrm{dG}, \mathrm{e}}$. To infer estimate (31), we resort to the $h p$-approximation properties stated in [19, Lemmas 23 and 33], implying

$$
\begin{aligned}
\mathcal{M}\left(\left(\dot{\boldsymbol{u}}-\dot{\boldsymbol{u}}_{I}, \dot{\boldsymbol{w}}-\dot{\boldsymbol{w}}_{I}, \dot{\varphi}-\right.\right. & \left.\left.\dot{\varphi}_{I}\right),\left(\dot{\boldsymbol{u}}-\dot{\boldsymbol{u}}_{I}, \dot{\boldsymbol{w}}-\dot{\boldsymbol{w}}_{I}, \dot{\varphi}-\dot{\varphi}_{I}\right)\right) \\
& \lesssim \sum_{\kappa \in \mathcal{T}_{h}^{p}}\left(\frac{h_{\kappa}^{2 s_{\kappa}}}{p_{p, \kappa}^{2 m}}\|\widetilde{\mathcal{E}} \dot{\boldsymbol{u}}\|_{m, \mathcal{K}_{\kappa}}^{2}+\frac{h_{\kappa}^{2 r_{\kappa}}}{p_{p, \kappa}^{2 \ell}}\|\widetilde{\mathcal{E}} \dot{\boldsymbol{w}}\|_{\ell, \mathcal{K}_{\kappa}}^{2}\right)+\sum_{\kappa \in \mathcal{T}_{h}^{a}} \frac{h_{\kappa}^{2 q_{\kappa}}}{p_{a, \kappa}^{2 n}}\|\widetilde{\mathcal{E}} \dot{\varphi}\|_{n, \mathcal{K}_{\kappa}}^{2},
\end{aligned}
$$


and, owing to (4.2),

$$
\mathcal{B}\left(\boldsymbol{w}-\boldsymbol{w}_{I}, \boldsymbol{w}-\boldsymbol{w}_{I}\right) \lesssim \sum_{\kappa_{p} \in \mathcal{T}_{h, p}^{I}} \frac{p_{p, \kappa_{p}}^{2}}{h_{\kappa_{p}}}\left\|\left(\boldsymbol{w}-\boldsymbol{w}_{I}\right) \cdot \boldsymbol{n}\right\|_{\partial \kappa_{p}}^{2} \lesssim \sum_{\kappa \in \mathcal{T}_{h}^{p}} \frac{h_{\kappa}^{2 r_{\kappa}-2}}{p_{p, \kappa}^{2 \ell-3}}\|\widetilde{\mathcal{E}} \boldsymbol{w}\|_{\ell, \mathcal{K}_{\kappa}}^{2} .
$$

We are now ready to state the main result of this section.

Theorem 3 (A-priori error estimates). Let Assumptions 3.1, 3.2, 4.1, and 4.2 hold and let the exact solution $\mathfrak{U}=(\boldsymbol{u}, \boldsymbol{w}, \varphi)$ of problem (8) be such that

$$
\left.\mathfrak{U} \in C^{2}\left([0, T] ; \boldsymbol{H}^{m}\left(\mathcal{T}_{h}^{p}\right) \times \boldsymbol{H}^{\ell}\left(\mathcal{T}_{h}^{p}\right)\right) \times H^{n}\left(\mathcal{T}_{h}^{a}\right)\right) \cap C^{1}\left([0, T] ; \boldsymbol{H}_{0}^{1}\left(\Omega_{p}\right) \times \boldsymbol{W}_{\tau} \times H_{0}^{1}\left(\Omega_{a}\right)\right),
$$

with $m, n, \ell \geq 2$ and let $\left(\boldsymbol{u}_{h}, \boldsymbol{w}_{h}, \varphi_{h}\right) \in C^{2}\left([0, T] ; \boldsymbol{V}_{h}^{p} \times \boldsymbol{V}_{h}^{p} \times V_{h}^{a}\right)$ be the solution of the semidiscrete problem (20), with sufficiently large penalty parameters $c_{1}, c_{2}$ and $c_{3}$. Then, for any $t \in(0, t]$, the discretization error $\boldsymbol{E}(t)=\left(\boldsymbol{e}^{u}, \boldsymbol{e}^{w}, e^{\varphi}\right)(t)$ satisfies

$$
\begin{aligned}
\|\boldsymbol{E}(t)\|_{\mathrm{E}} & \lesssim \sum_{\kappa \in \mathcal{T}_{h}^{p}} \frac{h_{\kappa}^{s_{\kappa}-1}}{p_{p, \kappa}^{m-3 / 2}}\left(\|\widetilde{\mathcal{E}} \dot{\boldsymbol{u}}\|_{m, \mathcal{K}_{\kappa}}+\|\widetilde{\mathcal{E}} \boldsymbol{u}\|_{m, \mathcal{K}_{\kappa}}+\int_{0}^{t}\left[\|\widetilde{\mathcal{E}} \ddot{\boldsymbol{u}}\|_{m, \mathcal{K}_{\kappa}}+\|\widetilde{\mathcal{E}} \dot{\boldsymbol{u}}\|_{m, \mathcal{K}_{\kappa}}\right](s) d s\right) \\
& +\sum_{\kappa \in \mathcal{T}_{h}^{p}} \frac{h_{\kappa}^{r_{\kappa}-1}}{p_{p, \kappa}^{\ell-3 / 2}}\left(\|\widetilde{\mathcal{E}} \dot{\boldsymbol{w}}\|_{\ell, \mathcal{K}_{\kappa}}+\|\widetilde{\mathcal{E} \boldsymbol{w}}\|_{\ell, \mathcal{K}_{\kappa}}+\int_{0}^{t}\left[\|\widetilde{\mathcal{E}} \ddot{\boldsymbol{w}}\|_{\ell, \mathcal{K}_{\kappa}}+\|\widetilde{\mathcal{E}} \dot{\boldsymbol{w}}\|_{\ell, \mathcal{K}_{\kappa}}\right](s) d s\right) \\
& +\sum_{\kappa \in \mathcal{T}_{h}^{a}} \frac{h_{\kappa}^{q_{\kappa}-1}}{p_{a, \kappa}^{n-3 / 2}}\left(\|\widetilde{\mathcal{E}} \dot{\varphi}\|_{n, \mathcal{K}_{\kappa}}+\|\widetilde{\mathcal{E}} \varphi\|_{n, \mathcal{K}_{\kappa}}+\int_{0}^{t}\left[\|\widetilde{\mathcal{E}} \ddot{\varphi}\|_{n, \mathcal{K}_{\kappa}}+\|\widetilde{\mathcal{E}} \dot{\varphi}\|_{n, \mathcal{K}_{\kappa}}\right](s) d s\right),
\end{aligned}
$$

where the hidden constant depends on time $t$ and on the material properties, but is independent of the discretization parameters and of $\tau$.

Proof. For any time $t \in(0, T]$, let $\left(\boldsymbol{u}_{I}, \boldsymbol{w}_{I}, \varphi_{I}\right)(t) \in \boldsymbol{V}_{h}^{p} \times \boldsymbol{V}_{h}^{p} \times V_{h}^{a}$ be the interpolants defined in (2). We split the error as $\boldsymbol{E}(t)=\boldsymbol{E}_{I}(t)-\boldsymbol{E}_{h}(t)$, where

$$
\begin{aligned}
& \boldsymbol{E}_{I}(t)=\left(\boldsymbol{e}_{I}^{u}, \boldsymbol{e}_{I}^{w}, e_{I}^{\varphi}\right)(t)=\left(\boldsymbol{u}-\boldsymbol{u}_{I}, \boldsymbol{w}-\boldsymbol{w}_{I}, \varphi-\varphi_{I}\right)(t), \\
& \boldsymbol{E}_{h}(t)=\left(\boldsymbol{e}_{h}^{u}, \boldsymbol{e}_{h}^{w}, e_{h}^{\varphi}\right)(t)=\left(\boldsymbol{u}_{h}-\boldsymbol{u}_{I}, \boldsymbol{w}_{h}-\boldsymbol{w}_{I}, \varphi_{h}-\varphi_{I}\right)(t) .
\end{aligned}
$$

From the triangle inequality we have $\|\boldsymbol{E}(t)\|_{\mathrm{E}}^{2} \leq\left\|\boldsymbol{E}_{h}(t)\right\|_{\mathrm{E}}^{2}+\left\|\boldsymbol{E}_{I}(t)\right\|_{\mathrm{E}}^{2}$, and Lemma 2 can be used to bound the term $\left\|\boldsymbol{E}_{I}(t)\right\|_{\mathrm{E}}$. As for the term $\left\|\boldsymbol{E}_{h}(t)\right\|_{\mathrm{E}}$, by taking $(\boldsymbol{v}, \boldsymbol{\xi}, \psi)=\left(\dot{\boldsymbol{e}}_{h}^{u}, \dot{\boldsymbol{e}}_{h}^{w}, \dot{e}_{h}^{\varphi}\right) \in$ $\boldsymbol{V}_{h}^{p} \times \boldsymbol{V}_{h}^{p} \times V_{h}^{a}$ as test functions in (29), taking into account that $\boldsymbol{E}=\boldsymbol{E}_{I}-\boldsymbol{E}_{h}$, neglecting the coupling terms thanks to skew-symmetry and collecting a first time derivative, identity (29) can be rewritten as

$$
\begin{aligned}
\frac{1}{2} \frac{d}{d t}\left(\mathcal{M}\left(\dot{\boldsymbol{E}}_{h}, \dot{\boldsymbol{E}}_{h}\right)+\right. & \left.\mathcal{A}_{h}\left(\boldsymbol{E}_{h}, \boldsymbol{E}_{h}\right)\right)+\mathcal{B}\left(\dot{\boldsymbol{e}}_{h}^{w}, \dot{\boldsymbol{e}}_{h}^{w}\right)=\mathcal{M}\left(\ddot{\boldsymbol{E}}_{I}, \dot{\boldsymbol{E}}_{h}\right)-\mathcal{A}_{h}\left(\dot{\boldsymbol{E}}_{I}, \boldsymbol{E}_{h}\right) \\
& +\frac{d}{d t} \mathcal{A}_{h}\left(\boldsymbol{E}_{I}, \boldsymbol{E}_{h}\right)+\mathcal{B}\left(\dot{\boldsymbol{e}}_{I}^{w}, \dot{\boldsymbol{e}}_{h}^{w}\right)+\mathcal{C}_{h}^{p}\left(\dot{e}_{I}^{\varphi}, \dot{\boldsymbol{e}}_{h}^{u}+\dot{\boldsymbol{e}}_{h}^{w}\right)+\mathcal{C}_{h}^{a}\left(\dot{\boldsymbol{e}}_{I}^{u}+\dot{\boldsymbol{e}}_{I}^{w}, \dot{e}_{h}^{\varphi}\right)
\end{aligned}
$$

where we have used Leibniz's rule on the term $\mathcal{A}_{h}\left(\boldsymbol{E}_{I}, \dot{\boldsymbol{E}}_{h}\right)$. Integrating (32) between 0 and $t \leq T$ and observing that $\boldsymbol{E}_{h}(0)=\left(\boldsymbol{e}_{h}^{u}(0), \boldsymbol{e}_{h}^{w}(0), e_{h}^{\varphi}(0)\right)=\mathbf{0}$, it is inferred that

$$
\begin{aligned}
& \mathcal{M}\left(\dot{\boldsymbol{E}}_{h}, \dot{\boldsymbol{E}}_{h}\right)(t)+\mathcal{A}_{h}\left(\boldsymbol{E}_{h}, \boldsymbol{E}_{h}\right)(t)+2 \int_{0}^{t} \mathcal{B}\left(\dot{\boldsymbol{e}}_{h}^{w}, \dot{\boldsymbol{e}}_{h}^{w}\right)(s) d s \\
& =2 \int_{0}^{t} \mathcal{M}\left(\ddot{\boldsymbol{E}}_{I}, \dot{\boldsymbol{E}}_{h}\right)(s) d s-2 \int_{0}^{t} \mathcal{A}_{h}\left(\dot{\boldsymbol{E}}_{I}, \boldsymbol{E}_{h}\right)(s) d s+2 \int_{0}^{t} \mathcal{B}\left(\dot{\boldsymbol{e}}_{I}^{w}, \dot{\boldsymbol{e}}_{h}^{w}\right)(s) d s
\end{aligned}
$$




$$
+2 \mathcal{A}_{h}\left(\boldsymbol{E}_{I}, \boldsymbol{E}_{h}\right)(t)+2 \int_{0}^{t}\left(\mathcal{C}_{h}^{p}\left(\dot{e}_{I}^{\varphi}, \dot{\boldsymbol{e}}_{h}^{u}+\dot{\boldsymbol{e}}_{h}^{w}\right)(s)+\mathcal{C}_{h}^{a}\left(\dot{\boldsymbol{e}}_{I}^{u}+\dot{\boldsymbol{e}}_{I}^{w}, \dot{e}_{h}^{\varphi}\right)(s)\right) d s
$$

Applying the Cauchy-Schwarz and Young inequalities on the third and fourth terms in the right-hand side of the previous identity, we obtain

$$
\begin{aligned}
1 & =\mathcal{M}\left(\dot{\boldsymbol{E}}_{h}, \dot{\boldsymbol{E}}_{h}\right)(t)+\mathcal{A}_{h}\left(\boldsymbol{E}_{h}, \boldsymbol{E}_{h}\right)(t)+\int_{0}^{t} \mathcal{B}\left(\dot{\boldsymbol{e}}_{h}^{w}, \dot{\boldsymbol{e}}_{h}^{w}\right)(s) d s \\
& \leq 4 \int_{0}^{t} \mathcal{M}\left(\ddot{\boldsymbol{E}}_{I}, \dot{\boldsymbol{E}}_{h}\right)(s) d s-4 \int_{0}^{t} \mathcal{A}_{h}\left(\dot{\boldsymbol{E}}_{I}, \boldsymbol{E}_{h}\right)(s) d s+2 \int_{0}^{t} \mathcal{B}\left(\dot{\boldsymbol{e}}_{I}^{w}, \dot{\boldsymbol{e}}_{I}^{w}\right)(s) d s \\
& +4 \mathcal{A}_{h}\left(\boldsymbol{E}_{I}, \boldsymbol{E}_{I}\right)(t)+4 \int_{0}^{t}\left(\mathcal{C}_{h}^{p}\left(\dot{e}_{I}^{\varphi}, \dot{\boldsymbol{e}}_{h}^{u}+\dot{\boldsymbol{e}}_{h}^{w}\right)+\mathcal{C}_{h}^{a}\left(\dot{\boldsymbol{e}}_{I}^{u}+\dot{\boldsymbol{e}}_{I}^{w}, \dot{e}_{h}^{\varphi}\right)\right)(s) d s=(2)
\end{aligned}
$$

Now, using Lemma A.2 together with the fundamental theorem of calculus we estimate the left hand side as (1) $\geq\left(\mathcal{M}\left(\dot{\boldsymbol{E}}_{h}, \dot{\boldsymbol{E}}_{h}\right)+\mathcal{A}_{h}\left(\boldsymbol{E}_{h}, \boldsymbol{E}_{h}\right)+\mathcal{B}\left(\boldsymbol{e}_{h}^{w}, \boldsymbol{e}_{h}^{w}\right)\right)(t)=\left\|\boldsymbol{E}_{h}(t)\right\|_{\mathrm{E}}^{2}$. Plugging this into (33), using again the Young inequality and Lemma A.2 to bound the second and fourth terms in (2), and recalling definition (30), yields

$$
\begin{aligned}
\left\|\boldsymbol{E}_{h}(t)\right\|_{\mathrm{E}}^{2} & \leq 2 \int_{0}^{t}\left\|\boldsymbol{E}_{h}(s)\right\|_{\mathrm{E}}^{2}+\overbrace{\left(\mathcal{M}\left(\ddot{\boldsymbol{E}}_{I}, \ddot{\boldsymbol{E}}_{I}\right)+\left\|\dot{\boldsymbol{E}}_{I}\right\|_{\mathrm{dG}}^{2}+\mathcal{B}\left(\dot{\boldsymbol{e}}_{I}^{w}, \dot{\boldsymbol{e}}_{I}^{w}\right)\right)(s) d s}^{\left\|\dot{\boldsymbol{E}}_{I}(s)\right\|_{\mathrm{E}}^{2}} \\
& +4\left\|\boldsymbol{E}_{I}(t)\right\|_{\mathrm{dG}}^{2}+4 \int_{0}^{t}\left(\mathcal{C}_{h}^{p}\left(\dot{e}_{I}^{\varphi}, \dot{\boldsymbol{e}}_{h}^{u}+\dot{\boldsymbol{e}}_{h}^{w}\right)+\mathcal{C}_{h}^{a}\left(\dot{\boldsymbol{e}}_{I}^{u}+\dot{\boldsymbol{e}}_{I}^{w}, \dot{e}_{h}^{\varphi}\right)\right)(s) d s .
\end{aligned}
$$

Now, recalling the definitions of the coupling bilinear forms $\mathcal{C}_{h}^{p}$ and $\mathcal{C}_{h}^{a}$ and using the CauchySchwarz inequality followed by the trace-inverse inequality (19), we infer

$$
\begin{aligned}
\mathcal{C}_{h}^{p}\left(\dot{e}_{I}^{\varphi}, \dot{\boldsymbol{e}}_{h}^{u}+\dot{\boldsymbol{e}}_{h}^{w}\right) & \lesssim \sum_{F \in \mathcal{F}_{h}^{I}}\left\|\rho_{a} \dot{e}_{I}^{\varphi}\right\|_{F}\left\|\dot{\boldsymbol{e}}_{h}^{u}+\dot{\boldsymbol{e}}_{h}^{w}\right\|_{F} \lesssim \sum_{\kappa_{p} \in \mathcal{T}_{h, p}^{I}, \kappa_{a} \in \mathcal{T}_{h, a}^{I}}\left\|\dot{e}_{I}^{\varphi}\right\|_{\partial \kappa_{a}}\left\|\dot{\boldsymbol{e}}_{h}^{u}+\dot{\boldsymbol{e}}_{h}^{w}\right\|_{\partial \kappa_{p}} \\
& \lesssim \sum_{\kappa_{p} \in \mathcal{T}_{h, p}^{I}, \kappa_{a} \in \mathcal{T}_{h, a}^{I}} p_{p, \kappa_{p}} h_{\kappa_{p}}^{-1 / 2}\left\|\dot{e}_{I}^{\varphi}\right\|_{\partial \kappa_{a}}\left(\left\|\dot{\boldsymbol{e}}_{h}^{u}\right\|_{\Omega_{p}}+\left\|\dot{\boldsymbol{e}}_{h}^{w}\right\|_{\Omega_{p}}\right)
\end{aligned}
$$

where, to infer the last bound, we have also used Assumption 3.2. Therefore, we have

$$
\begin{aligned}
\int_{0}^{t} \mathcal{C}_{h}^{p}\left(\dot{e}_{I}^{\varphi}, \dot{\boldsymbol{e}}_{h}^{u}+\dot{\boldsymbol{e}}_{h}^{w}\right)(s) d s & \lesssim \int_{0}^{t}\left(\sum_{\kappa \in \mathcal{T}_{h, a}^{I}} p_{a, \kappa} h_{\kappa}^{-1 / 2}\left\|\dot{e}_{I}^{\varphi}(s)\right\|_{\partial \kappa}\right)\left(\left\|\dot{\boldsymbol{e}}_{h}^{u}\right\|_{\Omega_{p}}+\left\|\dot{\boldsymbol{e}}_{h}^{w}\right\|_{\Omega_{p}}\right)(s) d s \\
& \stackrel{\operatorname{def}}{=} \int_{0}^{t} \mathcal{I}_{h}^{a}\left(\dot{e}_{I}^{\varphi}(s)\right)\left(\left\|\dot{\boldsymbol{e}}_{h}^{u}(s)\right\|_{\Omega_{p}}+\left\|\dot{\boldsymbol{e}}_{h}^{w}(s)\right\|_{\Omega_{p}}\right) d s .
\end{aligned}
$$

Proceeding in the same way, we can conclude that

$$
\begin{aligned}
\int_{0}^{t} \mathcal{C}_{h}^{a}\left(\dot{\boldsymbol{e}}_{I}^{u}+\dot{\boldsymbol{e}}_{I}^{w}, \dot{e}_{h}^{\varphi}\right)(s) d s & \lesssim \int_{0}^{t}\left(\sum_{\kappa \in \mathcal{T}_{h, p}^{I}} p_{p, \kappa} h_{\kappa}^{-1 / 2}\left(\left\|\dot{\boldsymbol{e}}_{I}^{u}\right\|_{\partial \kappa}+\left\|\dot{\boldsymbol{e}}_{I}^{w}\right\|_{\partial \kappa}\right)(s)\right)\left\|\dot{e}_{h}^{\varphi}(s)\right\|_{\Omega_{a}} d s \\
& \stackrel{\operatorname{def}}{=} \int_{0}^{t}\left(\mathcal{I}_{h}^{p}\left(\dot{\boldsymbol{e}}_{I}^{u}(s)\right)+\mathcal{I}_{h}^{p}\left(\dot{\boldsymbol{e}}_{I}^{w}(s)\right)\right)\left\|\dot{e}_{h}^{\varphi}(s)\right\|_{\Omega_{a}} d s,
\end{aligned}
$$

Collecting the two previous bounds and applying Young's inequality together with inequality (15), it is inferred that 


$$
\begin{aligned}
\int_{0}^{t}\left(\mathcal{C}_{h}^{p}\left(\dot{e}_{I}^{\varphi}, \dot{\boldsymbol{e}}_{h}^{u}+\dot{\boldsymbol{e}}_{h}^{w}\right)+\mathcal{C}_{h}^{a}\left(\dot{\boldsymbol{e}}_{I}^{u}\right.\right. & \left.\left.+\dot{\boldsymbol{e}}_{I}^{w}, \dot{e}_{h}^{\varphi}\right)\right)(s) d s \\
& \lesssim \int_{0}^{t}\left\|\boldsymbol{E}_{h}(s)\right\|_{\mathrm{E}}^{2} d s+\int_{0}^{t}\left(\mathcal{I}_{h}^{a}\left(\dot{e}_{I}^{\varphi}\right)^{2}+\mathcal{I}_{h}^{p}\left(\dot{\boldsymbol{e}}_{I}^{u}\right)^{2}+\mathcal{I}_{h}^{p}\left(\dot{\boldsymbol{e}}_{I}^{w}\right)^{2}\right)(s) d s .
\end{aligned}
$$

Hence, plugging the previous bound into (34) and using Gronwall's Lemma, we get

$$
\left\|\boldsymbol{E}_{h}(t)\right\|_{\mathrm{E}}^{2} \lesssim\left\|\boldsymbol{E}_{I}(t)\right\|_{\mathrm{E}}^{2}+\int_{0}^{t}\left\|\dot{\boldsymbol{E}}_{I}(s)\right\|_{\mathrm{E}}^{2} d s+\int_{0}^{t}\left(\mathcal{I}_{h}^{a}\left(\dot{e}_{I}^{\varphi}\right)^{2}+\mathcal{I}_{h}^{p}\left(\dot{\boldsymbol{e}}_{I}^{u}\right)^{2}+\mathcal{I}_{h}^{p}\left(\dot{\boldsymbol{e}}_{I}^{w}\right)^{2}\right)(s) d s,
$$

To estimate the terms on the right hand side, we make use of Lemma 2 and the following bounds inferred from [19, Lemma 33]:

$$
\begin{gathered}
\mathcal{I}_{h}^{a}\left(\dot{e}_{I}^{\varphi}\right)^{2} \lesssim \sum_{\kappa \in \mathcal{T}_{h, I}^{a}} \frac{h_{\kappa}^{2 q_{\kappa}-2}}{p_{a, \kappa}^{2 n-3}}\|\widetilde{\mathcal{E}} \dot{\varphi}\|_{n, \mathcal{K}_{\kappa}}^{2} \\
\mathcal{I}_{h}^{p}\left(\dot{\boldsymbol{e}}_{I}^{u}\right)^{2}+\mathcal{I}_{h}^{p}\left(\dot{\boldsymbol{e}}_{I}^{w}\right)^{2} \lesssim \sum_{\kappa \in \mathcal{T}_{h, I}^{p}} \frac{h_{\kappa}^{2 s_{\kappa}-2}}{p_{p, \kappa}^{2 m-3}}\|\widetilde{\mathcal{E}} \dot{\boldsymbol{u}}\|_{m, \mathcal{K}_{\kappa}}^{2}+\sum_{\kappa \in \mathcal{T}_{h, I}^{p}} \frac{h_{\kappa}^{2 r_{\kappa}-2}}{p_{p, \kappa}^{2 \ell-3}}\|\widetilde{\mathcal{E}} \dot{\boldsymbol{w}}\|_{\ell, \mathcal{K}_{\kappa}}^{2} .
\end{gathered}
$$

As a result, the thesis follows.

Corollary 1. Under the hypotheses of Theorem 3, assume that $h \approx h_{\kappa}$ for any $\kappa \in \mathcal{T}_{h}^{p} \cup \mathcal{T}_{h}^{a}$, $p_{p, \kappa}=p$ for any $\kappa \in \mathcal{T}_{h}^{p}$ and $p_{a, \kappa}=q$ for any $\kappa \in \mathcal{T}_{h}^{a}$. Then, if $\boldsymbol{u} \in C^{2}\left([0, T] ; \boldsymbol{H}^{m}\left(\Omega_{p}\right)\right)$, $\boldsymbol{w} \in C^{2}\left([0, T] ; \boldsymbol{H}^{\ell}\left(\Omega_{p}\right)\right)$ and $\varphi \in C^{2}\left([0, T] ; H^{n}\left(\Omega_{a}\right)\right)$, with $m, \ell \geq p+1, n \geq q+1$ the error estimate of Theorem 3 reads

$$
\begin{aligned}
\|\boldsymbol{E}(t)\|_{\mathrm{E}} & \lesssim \frac{h^{p}}{p^{m-3 / 2}}\left(\|\widetilde{\mathcal{E}} \dot{\boldsymbol{u}}\|_{m, \mathcal{K}_{\kappa}}+\|\widetilde{\mathcal{E} u}\|_{m, \mathcal{K}_{\kappa}}+\int_{0}^{t}\left[\|\widetilde{\mathcal{E}} \ddot{\boldsymbol{u}}\|_{m, \mathcal{K}_{\kappa}}+\|\widetilde{\mathcal{E}} \dot{\boldsymbol{u}}\|_{m, \mathcal{K}_{\kappa}}\right](s) d s\right) \\
& +\frac{h^{p}}{p^{\ell-3 / 2}}\left(\|\widetilde{\mathcal{E}} \dot{\boldsymbol{w}}\|_{\ell, \mathcal{K}_{\kappa}}+\|\widetilde{\mathcal{E} \boldsymbol{w}}\|_{\ell, \mathcal{K}_{\kappa}}+\int_{0}^{t}\left[\|\widetilde{\mathcal{E}} \ddot{\boldsymbol{w}}\|_{\ell, \mathcal{K}_{\kappa}}+\|\widetilde{\mathcal{E}} \dot{\boldsymbol{w}}\|_{\ell, \mathcal{K}_{\kappa}}\right](s) d s\right) \\
& +\frac{h^{q}}{q^{n-3 / 2}}\left(\|\widetilde{\mathcal{E}} \dot{\varphi}\|_{n, \mathcal{K}_{\kappa}}+\|\widetilde{\mathcal{E}} \varphi\|_{n, \mathcal{K}_{\kappa}}+\int_{0}^{t}\left[\|\widetilde{\mathcal{E}} \ddot{\varphi}\|_{n, \mathcal{K}_{\kappa}}+\|\widetilde{\mathcal{E}} \dot{\varphi}\|_{n, \mathcal{K}_{\kappa}}\right](s) d s\right),
\end{aligned}
$$

where the hidden constant depends on time $t$ and on the material properties, but is independent of the discretization parameters and $\tau$. The above bounds are optimal in $h$ and suboptimal in $p$ and $q$ by a factor $\frac{1}{2}$, see [40].

\section{Time discretization}

To integrate in time equation (26), we first discretize the interval $[0, T]$ by introducing a timestep $\Delta t>0$, such that $\forall k \in \mathbb{N}, t_{k+1}-t_{k}=\Delta t$ and define $\boldsymbol{X}^{k}$ as $\boldsymbol{X}^{k}=\boldsymbol{X}\left(t^{k}\right)$, with $\boldsymbol{X}=[U, W, \Phi]^{T}$. Next, we rewrite equation (26) in compact form as $\boldsymbol{A} \ddot{\boldsymbol{X}}+\boldsymbol{B} \dot{\boldsymbol{X}}+\boldsymbol{C} \boldsymbol{X}=\boldsymbol{F}$ and get

$$
\ddot{\boldsymbol{X}}=\boldsymbol{A}^{-1}(\boldsymbol{F}-\boldsymbol{B} \dot{\boldsymbol{X}}-\boldsymbol{C} \boldsymbol{X})=\boldsymbol{A}^{-1} \boldsymbol{F}-\boldsymbol{A}^{-1} \boldsymbol{B} \dot{\boldsymbol{X}}-\boldsymbol{A}^{-1} \boldsymbol{C} \boldsymbol{X}=\mathcal{L}(t, \boldsymbol{X}, \dot{\boldsymbol{X}}),
$$

Finally, to integrate in time (35) we can apply the Newmark- $\beta$ or the leap-frog scheme as follows. The Newmark $-\beta$ scheme is defined by introducing a Taylor expansion for displacement and velocity, respectively:

$$
\left\{\begin{array}{l}
\boldsymbol{X}^{k+1}=\boldsymbol{X}^{k}+\Delta t \boldsymbol{Z}^{k}+\Delta t^{2}\left(\beta_{N} \mathcal{L}^{k+1}+\left(\frac{1}{2}-\beta_{N}\right) \mathcal{L}^{k}\right) \\
\boldsymbol{Z}^{k+1}=\boldsymbol{Z}^{k}+\Delta t\left(\gamma_{N} \mathcal{L}^{k+1}+\left(1-\gamma_{N}\right) \mathcal{L}^{k}\right)
\end{array}\right.
$$


where $\boldsymbol{Z}^{k}=\dot{\boldsymbol{X}}\left(t^{k}\right), \mathcal{L}^{k}=\mathcal{L}\left(t^{k}, \boldsymbol{X}^{k}, \boldsymbol{Z}^{k}\right)$ and the Newmark parameters $\beta_{N}$ and $\gamma_{N}$ satisfy, the following constraints $0 \leq \gamma_{N} \leq 1,0 \leq 2 \beta_{N} \leq 1$. The typical choices of parameters are $\gamma_{N}=1 / 2$ and $\beta_{N}=1 / 4$, for which the scheme is unconditionally stable and second order accurate. Finally, by plugging the definition of $\mathcal{L}$ into (36), for $k \geq 0$, the time integration reduces to:

$$
\begin{array}{r}
{\left[\begin{array}{cc}
\boldsymbol{A}+\Delta t^{2} \beta_{N} \boldsymbol{C} & \Delta t^{2} \beta_{N} \boldsymbol{B} \\
\gamma_{N} \Delta t \boldsymbol{C} & \boldsymbol{A}+\gamma_{N} \Delta t \boldsymbol{B}
\end{array}\right]\left[\begin{array}{c}
\boldsymbol{X}^{k+1} \\
\boldsymbol{Z}^{k+1}
\end{array}\right]=\left[\begin{array}{cc}
\boldsymbol{A}-\Delta t^{2} \widetilde{\beta}_{N} \boldsymbol{C} & \Delta t \boldsymbol{A}-\Delta t^{2} \widetilde{\beta}_{N} \boldsymbol{B} \\
-\widetilde{\gamma}_{N} \Delta t \boldsymbol{C} & \boldsymbol{A}-\widetilde{\gamma}_{N} \Delta t \boldsymbol{B}
\end{array}\right]\left[\begin{array}{c}
\boldsymbol{X}^{k} \\
\boldsymbol{Z}^{k}
\end{array}\right]} \\
+\left[\begin{array}{c}
\Delta t^{2} \beta_{N} \boldsymbol{F}^{k+1}+\Delta t^{2} \widetilde{\beta}_{N} \boldsymbol{F}^{k} \\
\gamma_{N} \Delta t \boldsymbol{F}^{k+1}+\widetilde{\gamma}_{N} \Delta t \boldsymbol{F}^{k}
\end{array}\right],
\end{array}
$$

where $\widetilde{\beta}_{N}=\left(\frac{1}{2}-\beta_{N}\right)$ and $\widetilde{\gamma}_{N}=\left(1-\gamma_{N}\right)$. By applying the leap-frog scheme to (35) we get

$$
\left(\boldsymbol{A}+\frac{\Delta t^{2}}{2} \boldsymbol{B}\right) \boldsymbol{X}^{k+1}=\Delta t^{2} \boldsymbol{F}^{k}+\left(2 \boldsymbol{A}-\Delta t^{2} \boldsymbol{C}\right) \boldsymbol{X}^{k}+\left(\frac{\Delta t}{2} \boldsymbol{B}-\boldsymbol{A}\right) \boldsymbol{X}^{k-1},
$$

for $k \geq 1$ with initial step

$$
\boldsymbol{A} \boldsymbol{X}^{1}=\left(\boldsymbol{A}-\frac{\Delta t^{2}}{2} C\right) \boldsymbol{X}^{0}+\left(\Delta t \boldsymbol{A}-\frac{\Delta t^{2}}{2} \boldsymbol{B}\right) \boldsymbol{Z}^{0}+\frac{\Delta t^{2}}{2} \boldsymbol{F}^{0}
$$

Recall that (37)-(38) is explicit and second order accurate.

Remark 5.1. The leap-frog method is often applied to wave propagation problems due to its ease of implementation, the reduced size of the system (compared to a Newmark-type scheme), and because typically the matrix of the linear system to be solved is easily invertible. The latter in fact turns out to be diagonal or block-diagonal when using a dG method for the approximation in space. We note that in equation (37) this does not occur due to the coupling conditions at the interface between the poro-elastic and acoustic domains. As a further constraint, the fact that in poroelastic-acoustic materials there is an additional compressional wave of second kind (slow $\mathrm{P}$-wave) to be correctly propagated has an impact on the time integration scheme. Indeed, as a further outcome of the model, the amplitudes of the wavefield are attenuated because of energy loss due to the presence of a viscous fluid. In the case of low frequencies and a viscous fluid, the wave equations become stiff. In other words, the slow P-wave becomes the diffusive mode, which dominates the character of the equation and drastically restricts the stability condition for explicit methods. For these reasons we prefer to use an implicit time scheme, cf. also [23, 25].

\section{$6 \quad$ Numerical results}

Numerical implementation has been carried out with MATLAB. Meshes have been generated through the polymesher software, cf. [48].

\section{Test case 1}

The model problem is solved in $\Omega=(-1,1) \times(0,1)$, on a sequence of polygonal meshes as the one shown in Figure 2, and with physical parameters shown in Table 1. For the first test case, we choose as exact solution

$$
\begin{aligned}
& \boldsymbol{u}(x, y ; t)=\left(\begin{array}{l}
x^{2} \cos \left(\frac{\pi x}{2}\right) \sin (\pi x) \\
x^{2} \cos \left(\frac{\pi x}{2}\right) \sin (\pi x)
\end{array}\right) \cos (\sqrt{2} \pi t), \quad \boldsymbol{w}(x, y ; t)=-\boldsymbol{u}(x, y ; t), \\
& \varphi(x, y ; t)=\left(x^{2} \sin (\pi x) \sin (\pi y)\right) \sin (\sqrt{2} \pi t),
\end{aligned}
$$

in order to have a null pressure in the whole poroelastic domain. Since the solution together with its first $x-, y-$ and $t$ - derivatives are identically zero at the interface $\Gamma=0 \times(0,1)$, interface 


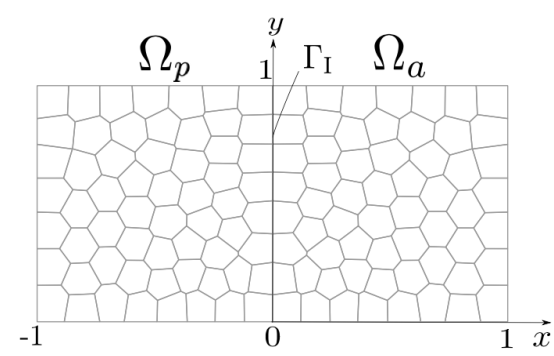

Figure 2: Test case 1. Polygonal mesh, with $N=100$ polygons.

\begin{tabular}{|c|c|}
\hline Field & Value \\
\hline$\rho_{f}, \rho_{s}$ & 1 \\
\hline$\lambda, \mu$ & 1 \\
\hline$a$ & 1 \\
\hline$\phi$ & 0.5 \\
\hline$\eta$ & 0 \\
\hline$\rho_{w}$ & 2 \\
\hline$\beta, \mathrm{m}$ & 1 \\
\hline$c, \rho_{a}$ & 1 \\
\hline
\end{tabular}

Table 1: Test case 1. Physical parameters.

coupling conditions are consequently null. This suggests to test the sealed pores $(\tau=0)$, the imperfect pores $(\tau \in(0,1))$ and the open pores $(\tau=1)$ cases with the same manufactured solution. A sequence of uniformly refined polygonal meshes have been considered, with uniform polynomial degree $p_{p, \kappa}=p_{a, \kappa}=p=1,2,3$. The final time $T$ has been set equal to 0.25 , considering a timestep of $\Delta t=10^{-4}$ for the Newmark- $\beta$ scheme, $\gamma_{N}=1 / 2$ and $\beta_{N}=1 / 4$. The penalty parameters $c_{1}, c_{2}$ and $c_{3}$ appearing in the definition (23)-(25) have been chosen equal to 10. In Figure 3 (left) we report the computed errors as a function of the inverse of the mesh-size ( $\log -\log$ scale), for the case $p=3$. As predicted by Theorem 3 the errors decays proportionally to $h^{3}$. Moreover, we have also computed the $L^{2}$-errors on the pressure field $p$. These results are reported Figure 4 and show a convergence rate proportional to $h^{3}$, as expected. We point out the that discrete pressure has been computed through equation (2). Finally, we compute the $L^{2}$ norm of the error fixing a computational mesh of $N=100$ polygons and varying the polynomial degree $p=1,2, \ldots, 5$. The computed errors are reported in Figure 3 (right) (semi-log scale), and an exponential decay of the error is clearly attained.

\section{Test case 2. Oblique interface}

The second test cases consider a domain $\Omega=(0,400) \times(0,400) \mathrm{m}^{2}$, with a straight interface with slope $60^{\circ}$, cf. Figure 5a. Physical and dimensional parameters have been chosen as in [23] and listed in Table 2.

\begin{tabular}{lllll}
\hline Fluid & Fluid density & $\rho_{f}, \rho_{a}$ & 1000 & $\mathrm{~kg} / \mathrm{m}^{3}$ \\
& Wave velocity & $c$ & 1500 & $\mathrm{~m} / \mathrm{s}$ \\
& Dynamic viscosity & $\eta$ & 0 & $\mathrm{~Pa} \cdot \mathrm{s}$ \\
\hline Grain & Solid density & $\rho_{s}$ & 2690 & $\mathrm{~kg} / \mathrm{m}^{3}$ \\
& Shear modulus & $\mu$ & $1.86 \cdot 10^{9}$ & $\mathrm{~Pa}$ \\
\hline Matrix & Porosity & $\phi$ & 0.38 & \\
& Tortuosity & $a$ & 1.8 & \\
& Permeability & $k$ & $2.79 \cdot 10^{-11}$ & $\mathrm{~m}^{2}$ \\
& Lamé coefficient & $\lambda$ & $1.2 \cdot 10^{8}$ & $\mathrm{~Pa}$ \\
& Biot's coefficient & $m$ & $5.34 \cdot 10^{9}$ & $\mathrm{~Pa}$ \\
& Biot's coefficient & $\beta$ & 0.95 & \\
\hline Interface & Interface permeability & $\tau$ & $\left\{0 ; 10^{-8} ; 1\right\}$ & \\
\hline
\end{tabular}

Table 2: Test case 2. Physical parameters.

Boundary and initial conditions have been set equal to zero both for the poroelastic and the acoustic domain. Forcing terms are null in $\Omega_{p}$, while in $\Omega_{a}$ a forcing term is imposed until 
Sealed pores $(\tau=0)$

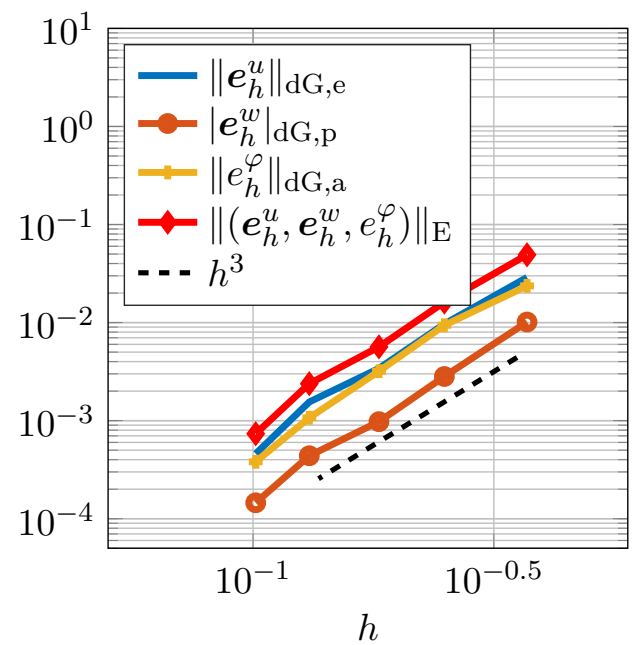

Imperfect pores $\left(\tau=\frac{1}{2}\right)$

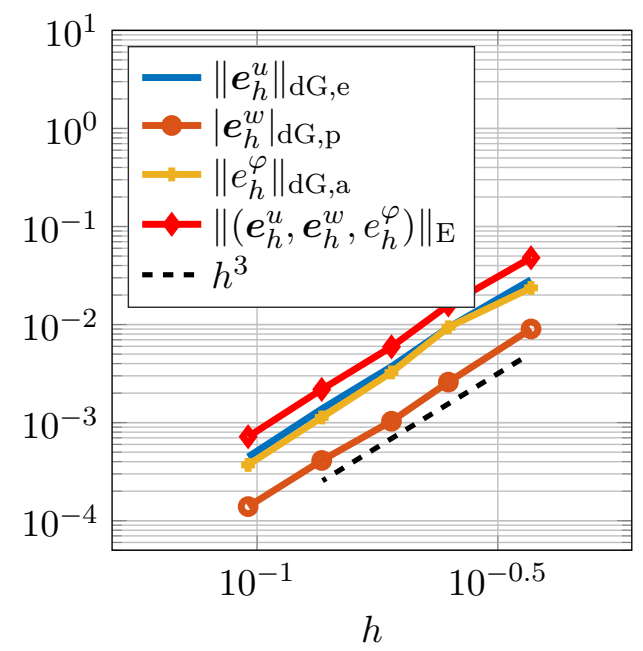

Open pores $(\tau=1)$

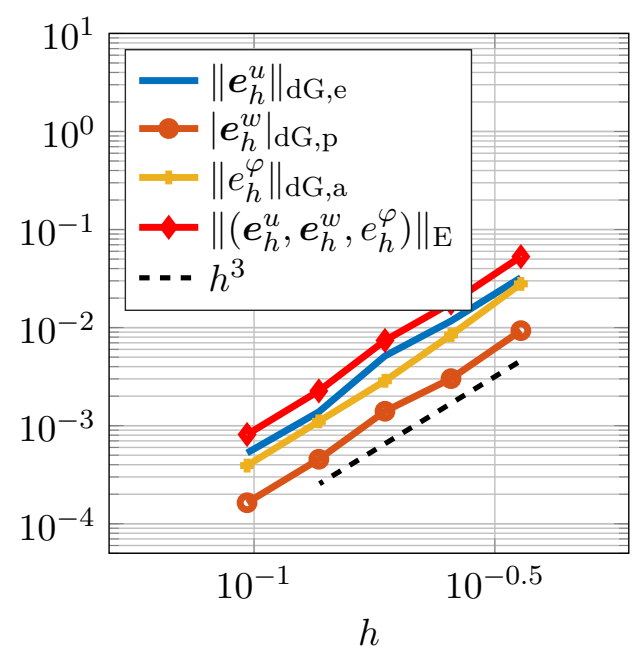

Sealed pores $(\tau=0)$

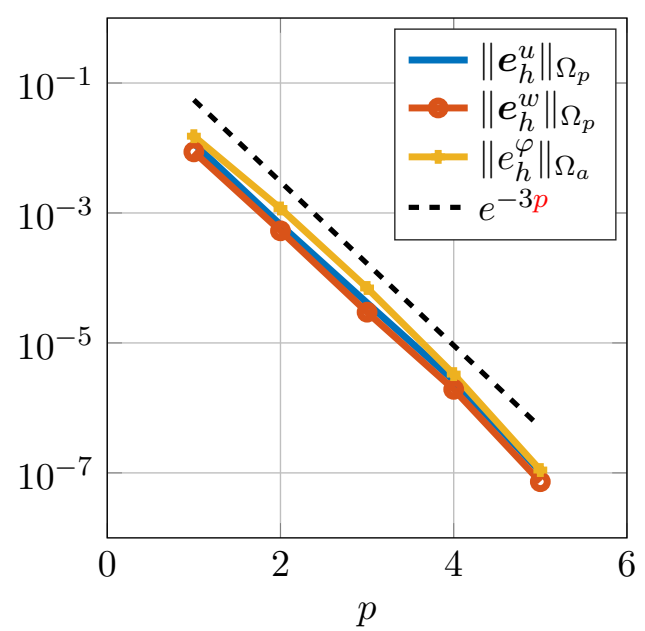

Imperfect pores $\left(\tau=\frac{1}{2}\right)$

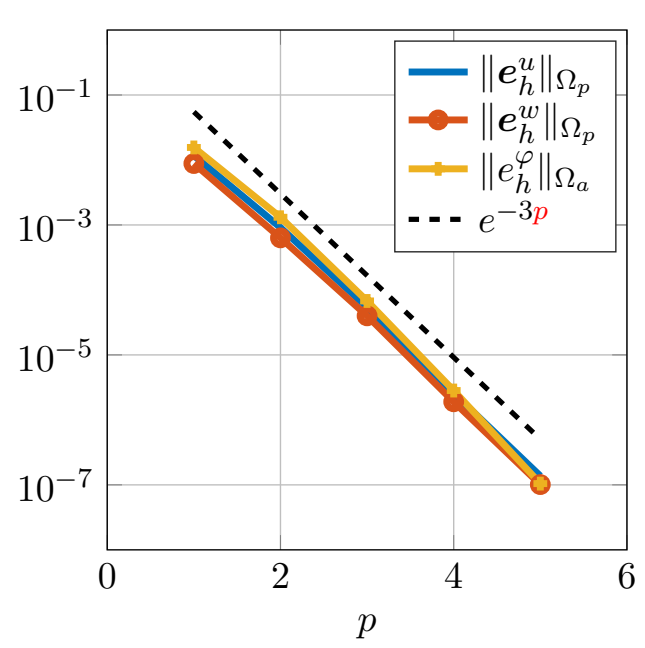

Open pores $(\tau=1)$

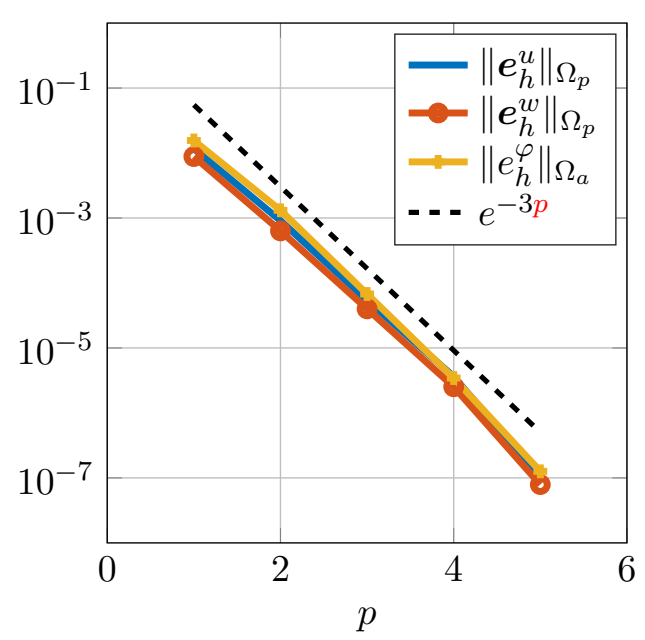

Figure 3: Test case 1. Left: computed errors in the energy norm, at the final time $T$, as a function of $h(p=3)$. Right: Computed errors in the $L^{2}$-norm, at final time $\mathrm{T}$, as a function of the polynomial degree $p$ on a computational mesh of $N=100$ polygons. 


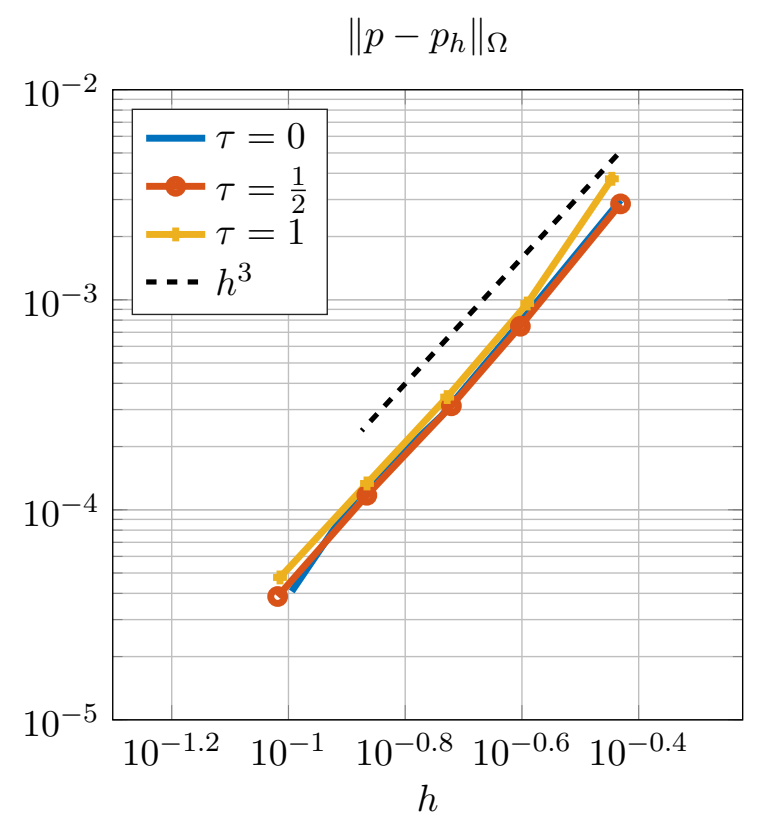

Figure 4: Test case 1. Computed errors $\left\|p-p_{h}\right\|_{\Omega}$, at the final time $T$, as a function of $h(p=3)$.

$t=0.05 \mathrm{~s}$, by considering the following load: $f_{a}=r(x, y) h(t)$, where

$$
h(t)= \begin{cases}\sum_{k=1}^{4} \alpha_{k} \sin \left(\gamma_{k} \omega_{0} t\right), & \text { if } 0<t<\frac{1}{f_{0}} \\ 0, & \text { otherwise }\end{cases}
$$

with coefficients defined as: $\alpha_{1}=1, \alpha_{2}=-21 / 32, \alpha_{3}=63 / 768, \alpha_{4}=-1 / 512, \gamma_{k}=2^{k-1}, \omega_{0}=$ $2 \pi f_{0} \mathrm{~Hz}, f_{0}=20 \mathrm{~Hz}$. The function $r(x, y)$ is defined as $r(x, y)=1$, if $(x, y) \in \bigcup_{i=1}^{4} B\left(\mathbf{x}_{i}, R\right)$, while $r(x, y)=0$, otherwise, where $B\left(\mathbf{x}_{i}, R\right)$ is the circle centered in $\mathbf{x}_{i}$ and with radius $R$. Here, we set $\mathbf{x}_{1}=(250,100) \mathrm{m}, \mathbf{x}_{2}=(250,150) \mathrm{m}, \mathbf{x}_{3}=(250,200) \mathrm{m}, \mathbf{x}_{4}=(250,250) \mathrm{m}$ and $R=10$ $\mathrm{m}$. Notice that, the support of the function $r(x, y)$ has been reported in Figure 5a, superimposed with a sample of one of the computational meshes employed. Simulations have been carried out by considering: a polygonal mesh consisting in $N=6586$ polygons, subdivided into $N_{a}=3564$ and $N_{p}=3022$ polygons for the acoustic and poroelastic domain, respectively; a Newmark scheme with time step $\Delta t=10^{-3} \mathrm{~s}$ and $\gamma_{N}=1 / 2$ and $\beta_{N}=1 / 4$ in a time interval $[0,0.15] \mathrm{s}$; a polynomial degree $p_{p, \kappa}=p_{a, \kappa}=p=4$. In Figure 6 , we show the computed pressure $p_{h}$ considering the interface permeability $\tau=0,10^{-8}$ and $\tau=1$, respectively. The latter values aim at modeling sealed, imperfect and open pores condition at the interface. Remark that $p_{h}=\rho_{a} \dot{\varphi}_{h}$ in the acoustic domain while $p_{h}=-m\left(\beta \nabla \cdot \boldsymbol{u}_{h}+\nabla \cdot \boldsymbol{w}_{h}\right)$ in the poroelastic one. As one can see, the pressure wave correctly propagates from the acoustic domain to the poroelastic one: the continuity at the interface boundary can be appreciated for the case $\tau=1$ (open pores).

\section{Test case 3: Sinusoidal interface}

Finally, with the same data of test case 2 , we consider a square domain $\Omega=[-1500,1500]^{2} \mathrm{~m}^{2}$ and a sinusoidal interface $\Gamma$ defined through the relation $\Gamma(x)=40 \sin \left(\frac{\pi}{100} x\right)$, cf. Figure 5b. For this numerical experiment we consider the dynamic viscosity $\eta=0$ and $\eta=0.0015$. The number of polygons composing the mesh is $N=5441$, subdivided into $N_{a}=2713$ and $N_{p}=2728$ polygons for the acoustic and poro-elastic subdomains, respectively. Moreover, as shown in Figure 5b, we have set the initial conditions on the acoustic domain, by defining $h(t)$ as before and $r(x, y)=1 / \rho_{a}$, if $(x, y) \in B\left(\mathbf{x}_{1}, R\right)$, and equal to 0 , otherwise, with $\mathbf{x}_{1}=(0,150) \mathrm{m}$ and $R=50 \mathrm{~m}$. Here we consider the interface permeability $\tau=1$. In Figure 7 we show the 


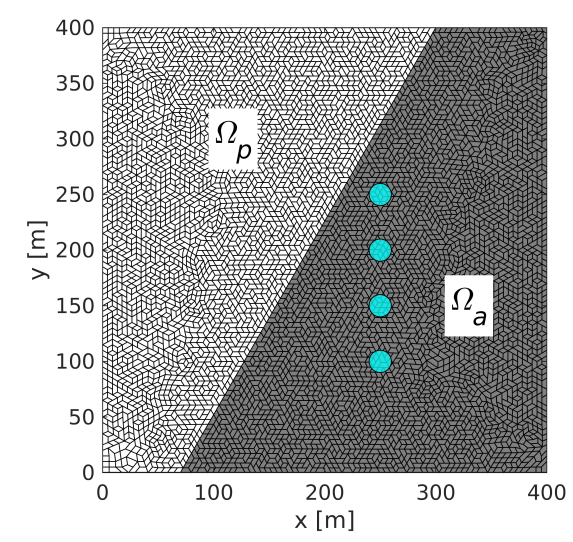

(a) Test case 2 .

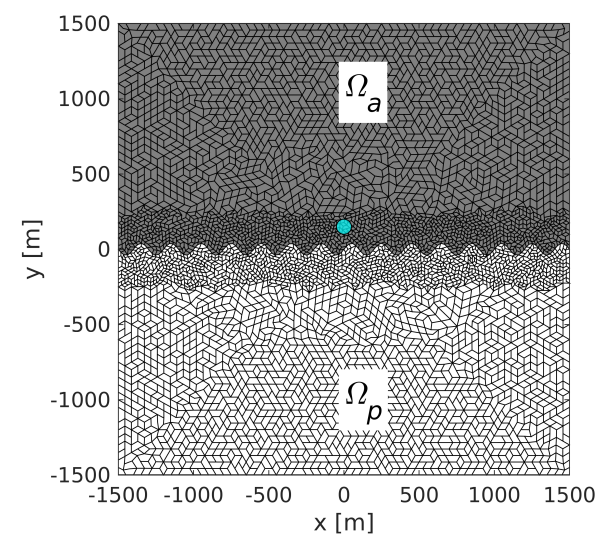

(b) Test case 3 .

Figure 5: Test cases 2 and 3. Computational domains and computational grids. The support of $\boldsymbol{r}(x, y)$ is also superimposed in cyan over the mesh.

propagation of the discrete pressure at the time instants $t=0.2,0.4 \mathrm{~s}$ and $t=0.6 \mathrm{~s}$. Observe how the sinusoidal interface contributes to the diffraction of the acoustic wave in the poroelastic domain. This effect is more relevant when the viscosity is null while for $\eta=0.0015$ the diffracted waves are attenuated in the poroelastic domain. In particular, we can observe the main wave front traveling towards the rigid walls of the domain followed by waves having smaller amplitude originated by the sinusoidal shape of the contact boundary.

\section{Conclusions}

In this work we have presented and analyzed a PolyDG approximation to the coupled poroelasto-acoustic problem on polygonal and polyhedral grids. Well-posedness of the continuous problem has been established by employing the semigroup theory. We a have proved a stability result for both the continuous and the semi-discrete formulations together with a priori $h p$ version error estimates for the semi-discrete solution in a suitable energy norm. Finally, a wide set of two-dimensional numerical simulations have been carried out.

\section{Acknowledgments}

PFA, MB and IM are members of the INdAM Research group GNCS and this work is partially funded by INdAM-GNCS. PFA has been partially funded by the research project PRIN17, n.201744KLJL funded by MIUR. The work of MB has been funded by the European Commission through the H2020-MSCA-IF-EF project PDGeoFF (Grant no. 896616). PA acknowledges the H2020-MSCA-IF-EF European Commission research grant no. 896616 (project PDGeoFF).

\section{A Theoretical results}

The existence and uniqueness of the solution to problem (8) as well as some technical results instrumental for the stability and error analysis are presented below.

We establish the existence and uniqueness result in the framework of the Hille-Yosida theory by combining and adapting the arguments of [4, Theorem 3.1] and [27, Section 5.2] where the elasto-acoustic coupling and the poroelastic problem were analyzed, respectively. To do so, we additionally define the spaces $\boldsymbol{H}_{\mathbb{C}}^{\Delta}\left(\Omega_{p}\right)=\left\{\boldsymbol{v} \in \boldsymbol{L}^{2}\left(\Omega_{p}\right): \nabla \cdot(\mathbb{C}: \boldsymbol{\epsilon}(\boldsymbol{v})) \in \boldsymbol{L}^{2}\left(\Omega_{p}\right)\right\}$, $\boldsymbol{H}^{\nabla}\left(\Omega_{p}\right)=\left\{\boldsymbol{v} \in \boldsymbol{L}^{2}\left(\Omega_{p}\right): \nabla(\nabla \cdot \boldsymbol{v}) \in \boldsymbol{L}^{2}\left(\Omega_{p}\right)\right\}$, and $H^{\Delta}\left(\Omega_{a}\right)=\left\{v \in L^{2}\left(\Omega_{a}\right): \Delta v \in L^{2}\left(\Omega_{a}\right)\right\}$. 

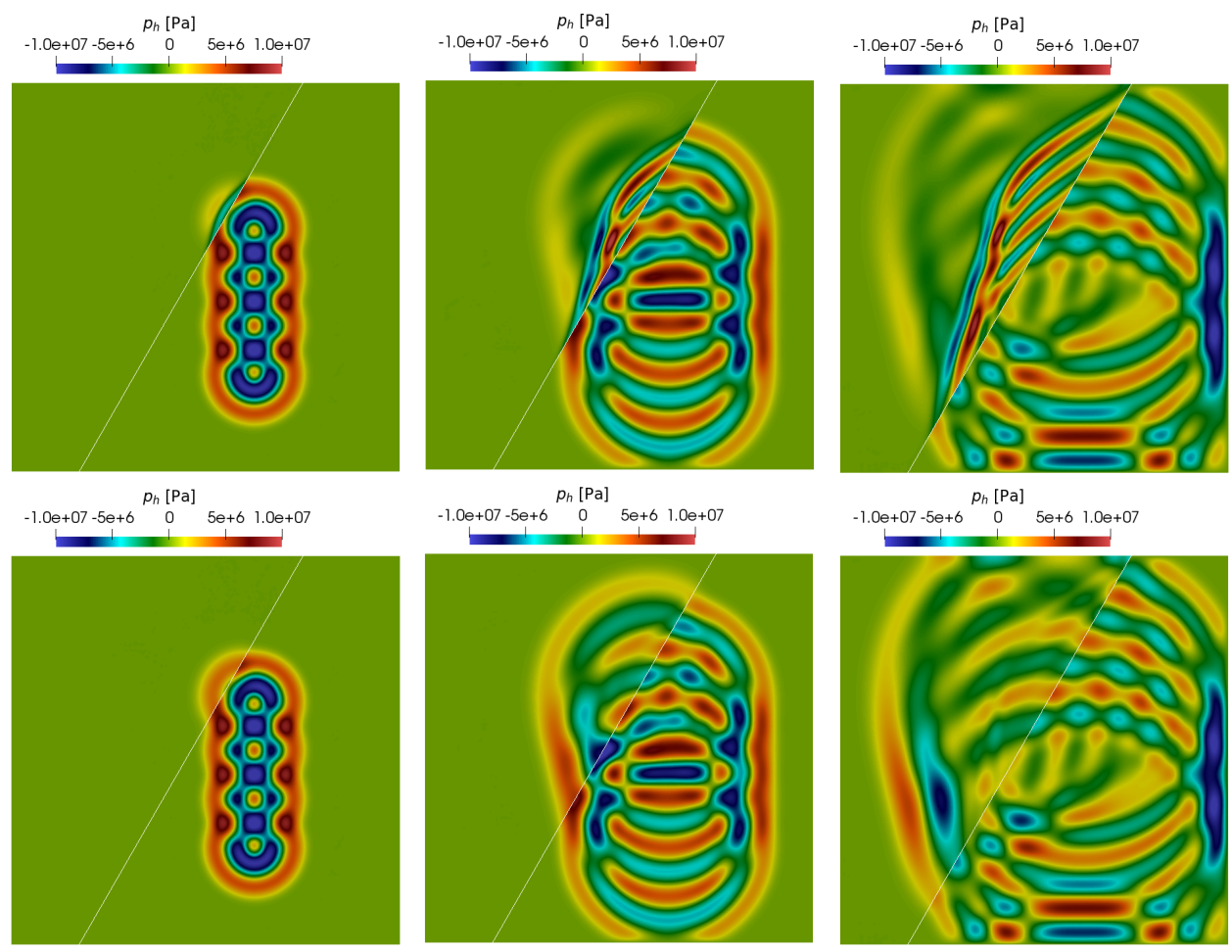

$-1.0 e+07-5 e+6 \stackrel{p_{h}[\mathrm{~Pa}]}{0} \quad 5 \mathrm{e}+6 \quad 1.0 \mathrm{e}+07$

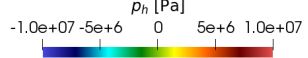

$-1.0 e+07-5 e+6 \stackrel{p_{h}[\mathrm{~Pa}]}{0} \quad 5 e+6 \quad 1.0 e+07$
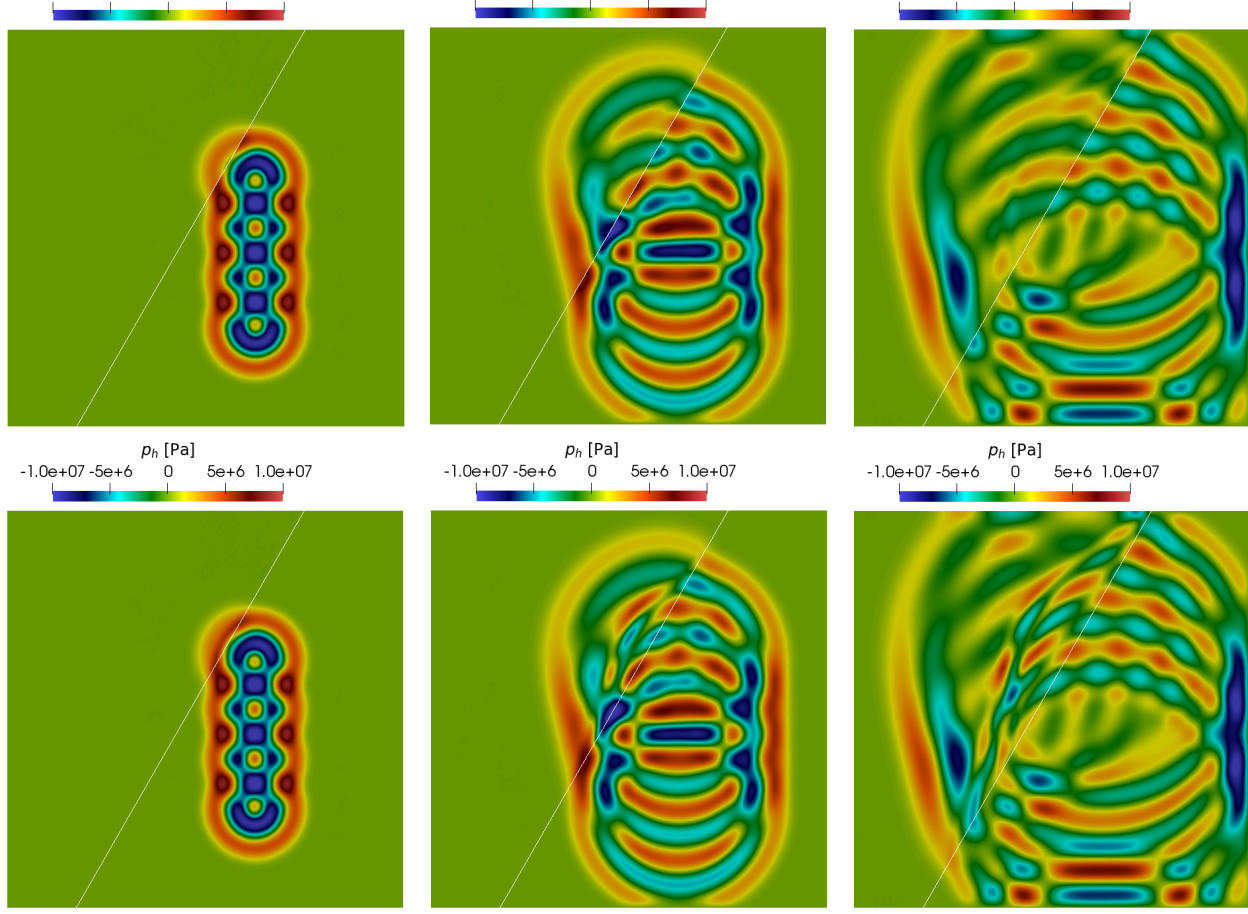

$-1.0 e+07-5 e+6 \stackrel{p_{h}[\mathrm{~Pa}]}{0} \quad 5 e+6 \quad 1.0 e+07$

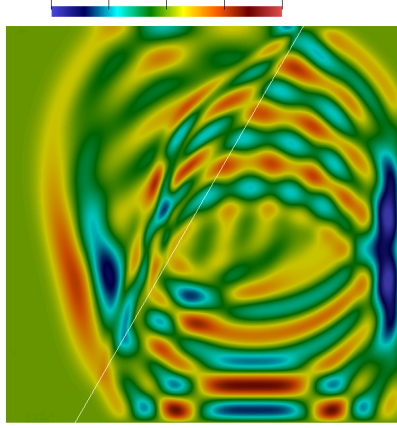

Figure 6: Test case 2. Oblique interface. Computed pressure $p_{h}$ in the poroelastic-acoustic domain at three time instants (from left to right $t=0.04,0.08,0.12 \mathrm{~s}$ ), with $\Delta t=10^{-3} \mathrm{~s}$. First line: $\tau=0$ (sealed pores). Second line: $\tau=10^{-8}$ (imperfect pores). Third line: $\tau=1$ (open pores). 

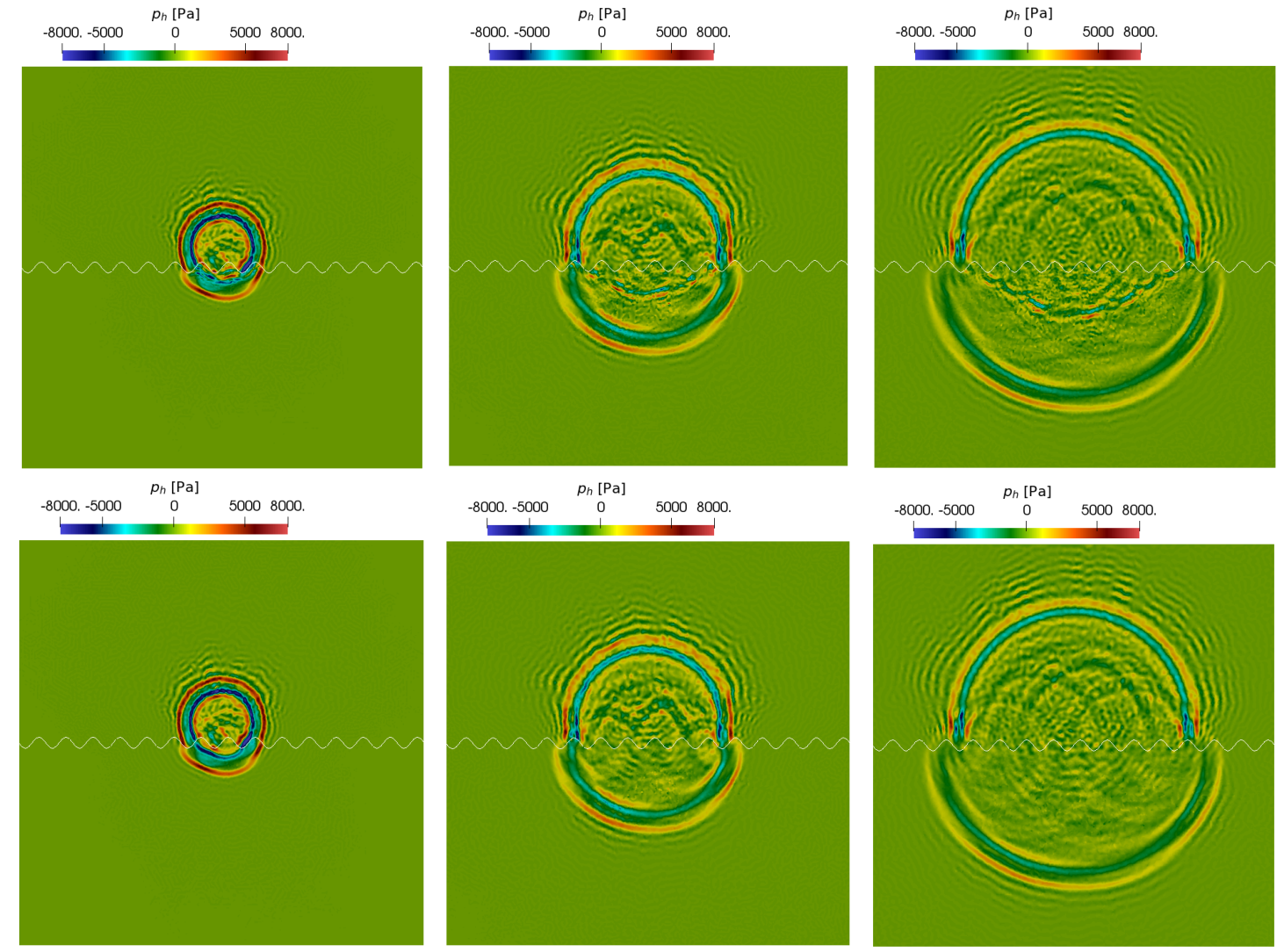

Figure 7: Test case 3. Computed pressure $p_{h}$ at the time instants $t=0.2 \mathrm{~s}$ (left), $t=0.4 \mathrm{~s}$ (center) and $t=0.6 \mathrm{~s}$ (right) with $\Delta t=10^{-3} \mathrm{~s}: \eta=0$ (top line) $\eta=0.0015$ (bottom line).

Theorem 4 (Existence and uniqueness of (8)). Assume that the initial data have the following regularity: $\boldsymbol{u}_{0} \in \boldsymbol{H}_{\mathbb{C}}^{\Delta}\left(\Omega_{p}\right) \cap \boldsymbol{H}_{0}^{1}\left(\Omega_{p}\right), \boldsymbol{u}_{1} \in \boldsymbol{H}_{0}^{1}\left(\Omega_{p}\right), \boldsymbol{w}_{0} \in \boldsymbol{W}_{\tau} \cap \boldsymbol{H}^{\nabla}\left(\Omega_{p}\right), \boldsymbol{w}_{1} \in \boldsymbol{W}_{\tau}, \varphi_{0} \in$ $H^{\Delta}\left(\Omega_{a}\right) \cap H_{0}^{1}\left(\Omega_{a}\right), \varphi_{1} \in H_{0}^{1}\left(\Omega_{a}\right)$, and that the source terms are such that $\boldsymbol{f}_{p} \in C^{1}\left([0, T] ; \boldsymbol{L}^{2}\left(\Omega_{p}\right)\right)$, $\boldsymbol{g}_{p} \in C^{1}\left([0, T] ; \boldsymbol{L}^{2}\left(\Omega_{p}\right)\right)$ and $f_{a} \in C^{1}\left([0, T] ; L^{2}\left(\Omega_{a}\right)\right)$. Then, problem (8) admits a unique strong solution $(\boldsymbol{u}, \boldsymbol{w}, \varphi)$ s.t.

$$
\begin{aligned}
& \boldsymbol{u} \in C^{2}\left([0, T] ; \boldsymbol{L}^{2}\left(\Omega_{p}\right)\right) \cap C^{1}\left([0, T] ; \boldsymbol{H}_{0}^{1}\left(\Omega_{p}\right)\right) \cap C^{0}\left([0, T] ; \boldsymbol{H}_{\mathbb{C}}^{\Delta}\left(\Omega_{p}\right) \cap \boldsymbol{H}_{0}^{1}\left(\Omega_{p}\right)\right), \\
& \boldsymbol{w} \in C^{2}\left([0, T] ; \boldsymbol{L}^{2}\left(\Omega_{p}\right)\right) \cap C^{1}\left([0, T] ; \boldsymbol{W}_{\tau}\right) \cap C^{0}\left([0, T] ; \boldsymbol{H}^{\nabla}\left(\Omega_{p}\right) \cap \boldsymbol{W}_{\tau}\right), \\
& \boldsymbol{\varphi} \in C^{2}\left([0, T] ; L^{2}\left(\Omega_{a}\right)\right) \cap C^{1}\left([0, T] ; H_{0}^{1}\left(\Omega_{a}\right)\right) \cap C^{0}\left([0, T] ; H^{\Delta}\left(\Omega_{a}\right) \cap H_{0}^{1}\left(\Omega_{a}\right)\right) .
\end{aligned}
$$

Proof. Let $\boldsymbol{v}=\dot{\boldsymbol{u}}, \boldsymbol{z}=\dot{\boldsymbol{w}}, \lambda=\dot{\varphi}$, and $\mathcal{U}=(\boldsymbol{u}, \boldsymbol{v}, \boldsymbol{w}, \boldsymbol{z}, \varphi, \lambda)$. We introduce the Hilbert space $\mathbb{V}=\boldsymbol{H}_{0}^{1}\left(\Omega_{p}\right) \times \boldsymbol{L}^{2}\left(\Omega_{p}\right) \times \boldsymbol{W}_{\tau} \times \boldsymbol{L}^{2}\left(\Omega_{p}\right) \times H_{0}^{1}\left(\Omega_{a}\right) \times L^{2}\left(\Omega_{a}\right)$, equipped with the scalar product

$$
\begin{aligned}
\left(\mathcal{U}_{1}, \mathcal{U}_{2}\right)_{\mathbb{V}}=\left(\rho \boldsymbol{v}_{1}+\rho_{f} \boldsymbol{z}_{1}, \boldsymbol{v}_{2}\right)_{\Omega_{p}}+\left(\rho_{f} \boldsymbol{v}_{1}+\rho_{w} \boldsymbol{z}_{1}, \boldsymbol{z}_{2}\right)_{\Omega_{p}}+\left(\rho_{a} c^{-2} \lambda_{1}, \lambda_{2}\right)_{\Omega_{a}} \\
+\left(\mathbb{C}: \boldsymbol{\epsilon}\left(\boldsymbol{u}_{1}\right), \boldsymbol{\epsilon}\left(\boldsymbol{u}_{2}\right)\right)_{\Omega_{p}}+\left(m \nabla \cdot\left(\beta \boldsymbol{u}_{1}+\boldsymbol{w}_{1}\right), \nabla \cdot\left(\beta \boldsymbol{u}_{2}+\boldsymbol{w}_{2}\right)\right)_{\Omega_{p}} \\
+\left(\rho_{a} \nabla \varphi_{1}, \nabla \varphi_{2}\right)_{\Omega_{a}}+\left(\eta k^{-1} \boldsymbol{w}_{1}, \boldsymbol{w}_{2}\right)_{\Omega_{p}}+\left(\zeta(\tau) \boldsymbol{w}_{1} \cdot \boldsymbol{n}_{p}, \boldsymbol{w}_{2} \cdot \boldsymbol{n}_{p}\right)_{\Gamma_{I}},
\end{aligned}
$$

where $\boldsymbol{W}_{\tau}$ is defined in (9). We remark that the scalar product is positive definite in $\mathbb{V} \times \mathbb{V}$, cf. [27]. We define the operator

$$
A: \mathcal{D}(A) \subset \mathbb{V} \rightarrow \mathbb{V} \quad A \mathcal{U}=\left(\begin{array}{c}
-\boldsymbol{v} \\
-\frac{1}{\rho_{T}}\left(\rho_{w} \nabla \cdot \boldsymbol{\sigma}+\frac{\rho_{f} \eta}{k} \boldsymbol{z}+\rho_{f} \nabla p\right) \\
-\boldsymbol{z} \\
\frac{1}{\rho_{T}}\left(\rho_{f} \nabla \cdot \boldsymbol{\sigma}+\frac{\rho \eta}{k} \boldsymbol{z}+\rho \nabla p\right) \\
-\lambda \\
-c^{2} \rho_{a}^{-1} \nabla \cdot\left(\rho_{a} \nabla \varphi\right)
\end{array}\right)
$$


with $\rho_{T}=\rho \rho_{w}-\rho_{f}^{2}>0$, and

$$
\begin{aligned}
& \mathcal{D}(A)=\left\{\mathcal{U} \in \mathbb{V}: \boldsymbol{u} \in \boldsymbol{H}_{\mathbb{C}}^{\Delta}\left(\Omega_{p}\right), \boldsymbol{v} \in \boldsymbol{H}_{0}^{1}\left(\Omega_{p}\right), \boldsymbol{w} \in \boldsymbol{H}^{\nabla}\left(\Omega_{p}\right), \boldsymbol{z} \in \boldsymbol{W}_{\tau},\right. \\
& \varphi \in H^{\Delta}\left(\Omega_{a}\right), \lambda \in H_{0}^{1}\left(\Omega_{a}\right) ;\left(\boldsymbol{\sigma}+\rho_{a} \lambda \boldsymbol{I}\right) \cdot \boldsymbol{n}_{p}=\mathbf{0}, \text { on } \Gamma_{I}, \\
&\left.\tau\left(p-\rho_{a} \lambda\right)-(1-\tau) \boldsymbol{z} \cdot \boldsymbol{n}_{p}=0, \text { on } \Gamma_{I},(\nabla \varphi+\boldsymbol{v}+\boldsymbol{z}) \cdot \boldsymbol{n}_{p}=0, \text { on } \Gamma_{I}\right\} .
\end{aligned}
$$

With the above notation, problem (8) can be reformulated as follows: given $\mathcal{F} \in C^{1}([0, T] ; \mathbb{V})$ defined as $\mathcal{F}(t)=\left(\mathbf{0},\left(\rho_{w} \boldsymbol{f}_{p}-\rho_{f} \boldsymbol{g}_{p}\right) / \rho_{T}, \mathbf{0},\left(\rho \boldsymbol{g}_{p}-\rho_{f} \boldsymbol{f}_{p}\right) / \rho_{T}, 0, c^{2} f_{a}\right)$ and $\mathcal{U}_{0} \in \mathcal{D}(A)$, find $\mathcal{U} \in$ $C^{1}([0, T] ; \mathbb{V}) \cap C^{0}([0, T] ; \mathcal{D}(A))$ such that

$$
\left\{\begin{array}{l}
\frac{\mathrm{d} \mathcal{U}}{\mathrm{dt}}+A \mathcal{U}(t)=\mathcal{F}(t), \quad t \in(0, T] \\
\mathcal{U}(0)=\mathcal{U}_{0}
\end{array}\right.
$$

Owing to the Hille-Yosida theorem, the above problem is well-posed provided the existence of $\mu>0$ such that $A+\mu I$ is maximal monotone, i.e. $(A \mathcal{U}, \mathcal{U})_{\mathbb{V}}+\mu\|\mathcal{U}\|_{\mathbb{V}}^{2} \geq 0 \forall \mathcal{U} \in \mathcal{D}(A)$ and $A+\mu I: \mathcal{D}(A) \rightarrow \mathbb{V}$ is onto. The first condition follows from the definition of the scalar product in $\mathbb{V}$, the definition of $\mathcal{D}(A)$ and integration by parts:

$$
\begin{aligned}
(A \mathcal{U}, \mathcal{U})_{\mathbb{V}}= & -\left(\frac{\rho \rho_{w}}{\rho_{T}} \nabla \cdot \boldsymbol{\sigma}+\frac{\rho \rho_{f}}{\rho_{T}} \frac{\eta}{k} \boldsymbol{z}+\frac{\rho \rho_{f}}{\rho_{T}} \nabla p, \boldsymbol{v}\right)_{\Omega_{p}}-(\mathbb{C}: \boldsymbol{\epsilon}(\boldsymbol{v}), \boldsymbol{\epsilon}(\boldsymbol{u}))_{\Omega_{p}} \\
& +\left(\frac{\rho_{f}^{2}}{\rho_{T}} \nabla \cdot \boldsymbol{\sigma}+\frac{\rho \rho_{f}}{\rho_{T}} \frac{\eta}{k} \boldsymbol{z}+\frac{\rho \rho_{f}}{\rho_{T}} \nabla p, \boldsymbol{v}\right)_{\Omega_{p}}-\left(\nabla \cdot \rho_{a} \nabla \varphi, \lambda\right)_{\Omega_{a}} \\
& -\left(\frac{\rho_{f} \rho_{w}}{\rho_{T}} \nabla \cdot \boldsymbol{\sigma}+\frac{\rho_{f}^{2}}{\rho_{T}} \frac{\eta}{k} \boldsymbol{z}+\frac{\rho_{f}^{2}}{\rho_{T}} \nabla p, \boldsymbol{z}\right)_{\Omega_{p}}-\left(\rho_{a} \nabla \lambda, \nabla \varphi\right)_{\Omega_{a}} \\
& +\left(\frac{\rho_{w} \rho_{f}}{\rho_{T}} \nabla \cdot \boldsymbol{\sigma}+\frac{\rho_{w} \rho}{\rho_{T}} \frac{\eta}{k} \boldsymbol{z}+\frac{\rho_{w} \rho}{\rho_{T}} \nabla p, \boldsymbol{z}\right)_{\Omega_{p}}-\left(\eta k^{-1} \boldsymbol{z}, \boldsymbol{w}\right)_{\Omega_{p}} \\
& -(m \nabla \cdot(\beta \boldsymbol{v}+\boldsymbol{z}), \nabla \cdot(\beta \boldsymbol{u}+\boldsymbol{w}))_{\Omega_{p}}-\left(\zeta(\tau) \boldsymbol{z} \cdot \boldsymbol{n}_{p}, \boldsymbol{w} \cdot \boldsymbol{n}_{p}\right)_{\Gamma_{I}} \\
& =\left\|(\eta / k)^{\frac{1}{2}} \boldsymbol{z}\right\|_{\Omega_{p}}^{2}+\left\|\zeta(\tau)^{\frac{1}{2}} \boldsymbol{z} \cdot \boldsymbol{n}_{p}\right\|_{\Gamma_{I}}^{2}-((\eta / k) \boldsymbol{z}, \boldsymbol{w})_{\Omega_{p}}-\left(\zeta(\tau) \boldsymbol{z} \cdot \boldsymbol{n}_{p}, \boldsymbol{w} \cdot \boldsymbol{n}_{p}\right)_{\Gamma_{I}},
\end{aligned}
$$

where we have also used that all the terms on $\Gamma_{I}$ (except $\left\|\zeta(\tau)^{1 / 2} \boldsymbol{z} \cdot \boldsymbol{n}_{p}\right\|_{\Gamma_{I}}^{2}$ for $\tau \in(0,1)$ ) vanish. Thus, by choosing $\mu \geq 1 / 2$, and applying the Young's inequality, we obtain $(A \mathcal{U}, \mathcal{U})_{\mathbb{V}}+\mu\|\mathcal{U}\|_{\mathbb{V}}^{2} \geq$ 0 . Now, we prove that $A+\nu I$ is surjective for all $\nu>0$. The surjectivity of $A+\nu I$ is equivalent to verify that for any $\mathcal{F} \in \mathbb{V}$, there exists $\mathcal{U} \in \mathcal{D}(A)$ s.t. $A \mathcal{U}+\nu \mathcal{U}=\mathcal{F}$, i.e.

$$
\begin{aligned}
& \nu \boldsymbol{u}-\boldsymbol{v}=\mathcal{F}_{1}, \\
& \nu \boldsymbol{v}-\frac{\rho_{w}}{\rho_{T}} \nabla \cdot \boldsymbol{\sigma}-\frac{\rho_{f}}{\rho_{T}} \frac{\eta}{k} \boldsymbol{z}-\frac{\rho_{f}}{\rho_{T}} \nabla p=\mathcal{F}_{2}, \\
& \nu \boldsymbol{w}-\boldsymbol{z}=\mathcal{F}_{3}, \\
& \nu \boldsymbol{z}+\frac{\rho_{f}}{\rho_{T}} \nabla \cdot \boldsymbol{\sigma}+\frac{\rho}{\rho_{T}} \frac{\eta}{k} \boldsymbol{z}+\frac{\rho}{\rho_{T}} \nabla p=\mathcal{F}_{4}, \\
& \nu \varphi-\lambda=\mathcal{F}_{5}, \\
& \nu \lambda-c^{2} \rho_{a}^{-1} \nabla \cdot\left(\rho_{a} \nabla \varphi\right)=\mathcal{F}_{6} .
\end{aligned}
$$

Hence, by plugging $\boldsymbol{v}=\nu \boldsymbol{u}-\mathcal{F}_{1}, \boldsymbol{z}=\nu \boldsymbol{w}-\mathcal{F}_{3}$, and $\lambda=\nu \varphi-\mathcal{F}_{5}$ respectively in (40b), (40d), and (40f) and rearranging, we rewrite the previous system as

$$
\left\{\begin{array}{l}
\nu^{2}\left(\rho \boldsymbol{u}+\rho_{f} \boldsymbol{w}\right)-\nabla \cdot \boldsymbol{\sigma}=\rho\left(\nu \mathcal{F}_{1}+\mathcal{F}_{2}\right)+\rho_{f}\left(\nu \mathcal{F}_{3}+\mathcal{F}_{4}\right)=\boldsymbol{G}_{1} \\
\nu^{2}\left(\rho_{f} \boldsymbol{u}+\rho_{w} \boldsymbol{w}\right)+\frac{\nu \eta}{k} \boldsymbol{w}+\nabla p=\rho_{f}\left(\nu \mathcal{F}_{1}+\mathcal{F}_{2}\right)+\rho_{w}\left(\nu \mathcal{F}_{3}+\mathcal{F}_{4}\right)+\frac{\eta}{k} \mathcal{F}_{3}=G_{2} \\
\nu^{2} \rho_{a} c^{-2} \varphi-\nabla \cdot\left(\rho_{a} \nabla \varphi\right)=\rho_{a} c^{-2}\left(\nu \mathcal{F}_{5}+\mathcal{F}_{6}\right)=G_{3}
\end{array}\right.
$$


Owing to $\boldsymbol{n}_{p}=-\boldsymbol{n}_{a}$ on $\Gamma_{I}$, equations (40a), (40c) and (40e), and the transmission conditions on $\Gamma_{I}$ embedded in the definition of $\mathcal{D}(A)$, the variational formulation of the above problem reads: find $(\boldsymbol{u}, \boldsymbol{w}, \varphi) \in \boldsymbol{H}_{0}^{1}\left(\Omega_{p}\right) \times \boldsymbol{W}_{\tau} \times H_{0}^{1}\left(\Omega_{a}\right)$ s.t.

$$
\mathcal{A}((\boldsymbol{u}, \boldsymbol{w}, \varphi),(\boldsymbol{v}, \boldsymbol{z}, \lambda))=\mathcal{L}(\boldsymbol{v}, \boldsymbol{z}, \lambda), \quad \text { for all }(\boldsymbol{v}, \boldsymbol{z}, \lambda) \in \boldsymbol{H}_{0}^{1}\left(\Omega_{p}\right) \times \boldsymbol{W}_{\tau} \times H_{0}^{1}\left(\Omega_{a}\right),
$$

with

$$
\begin{aligned}
\mathcal{A}((\boldsymbol{u}, \boldsymbol{w}, \varphi),(\boldsymbol{v}, \boldsymbol{z}, \lambda)) & =\nu^{2}\left(\rho \boldsymbol{u}+\rho_{f} \boldsymbol{w}, \boldsymbol{v}\right)_{\Omega_{p}}+(\mathbb{C} \boldsymbol{\epsilon}(\boldsymbol{u}), \boldsymbol{\epsilon}(\boldsymbol{v}))_{\Omega_{p}}+\nu^{2}\left(\rho_{f} \boldsymbol{u}+\rho_{w} \boldsymbol{w}, \boldsymbol{z}\right)_{\Omega_{p}} \\
& +(m \nabla \cdot(\beta \boldsymbol{u}+\boldsymbol{w}), \nabla \cdot(\beta \boldsymbol{v}+\boldsymbol{z}))_{\Omega_{p}}+\nu\left(\eta k^{-1} \boldsymbol{w}, \boldsymbol{z}\right)_{\Omega_{p}} \\
& +\nu\left(\zeta(\tau) \boldsymbol{w} \cdot \boldsymbol{n}_{p}, \boldsymbol{z} \cdot \boldsymbol{n}_{p}\right)_{\Gamma_{I}}+\nu^{2}\left(\rho_{a} c^{-2} \varphi, \lambda\right)_{\Omega_{a}} \\
& +\left(\rho_{a} \nabla \varphi, \nabla \lambda\right)_{\Omega_{a}}+\nu\left(\rho_{a} \varphi, \boldsymbol{v} \cdot \boldsymbol{n}_{p}\right)_{\Gamma_{I}}-\nu\left(\boldsymbol{u} \cdot \boldsymbol{n}_{p}, \rho_{a} \lambda\right)_{\Gamma_{I}} \\
\text { and } \quad \mathcal{L}(\boldsymbol{v}, \boldsymbol{z}, \lambda) & =\left(\boldsymbol{G}_{1}, \boldsymbol{v}\right)_{\Omega_{p}}+\left(\boldsymbol{G}_{2}, \boldsymbol{z}\right)_{\Omega_{p}}+\left(G_{3}, \lambda\right)_{\Omega_{a}}-\left(\mathcal{F}_{1} \cdot \boldsymbol{n}_{p}, \rho_{a} \lambda\right)_{\Gamma_{I}} \\
& +\left(\zeta(\tau) \mathcal{F}_{3} \cdot \boldsymbol{n}_{p}, \boldsymbol{z} \cdot \boldsymbol{n}_{p}\right)_{\Gamma_{I}}+\left(\rho_{a} \mathcal{F}_{5}, \boldsymbol{v} \cdot \boldsymbol{n}_{p}\right)_{\Gamma_{I}}
\end{aligned}
$$

The well-posedness of the previous problem follows from the Lax-Milgram Lemma, since $\mathcal{A}$ is coercive for all $\nu>0$. In addition, owing to (40b), (40d), and (40f), we infer that $\boldsymbol{u} \in$ $\boldsymbol{H}_{\mathbb{C}}^{\Delta}\left(\Omega_{p}\right) \cap \boldsymbol{H}_{0}^{1}\left(\Omega_{p}\right), \boldsymbol{w} \in \boldsymbol{H}^{\nabla}\left(\Omega_{p}\right) \cap \boldsymbol{W}_{\tau}$, and $\varphi \in H^{\Delta}\left(\Omega_{a}\right) \cap H_{0}^{1}\left(\Omega_{a}\right)$. Moreover, this gives $(\boldsymbol{v}, \boldsymbol{z}, \lambda) \in \boldsymbol{H}_{0}^{1}\left(\Omega_{p}\right) \times \boldsymbol{W}_{\tau} \times H_{0}^{1}\left(\Omega_{a}\right)$ due to (40a), (40c), and (40e). Then $\mathcal{U} \in \mathcal{D}(A)$ and the proof is complete.

We conclude the Appendix with some technical results needed in the analysis. The first Lemma hinges on Assumption 3.1 and the trace inverse inequality (19).

Lemma A.1. The following bounds hold:

$$
\begin{aligned}
\left\|\alpha^{-1 / 2}\left\{\boldsymbol{\sigma}_{h}(\boldsymbol{v})\right\}\right\|_{\mathcal{F}_{h}^{p}} & \lesssim \frac{1}{\sqrt{c_{1}}}\left\|\mathbb{C}^{1 / 2} \boldsymbol{\epsilon}_{h}(\boldsymbol{v})\right\|_{\Omega_{p}} & \forall \boldsymbol{v} \in \boldsymbol{V}_{h}^{p}, \\
\left\|\chi^{-1 / 2}\left\{\rho_{a} \nabla_{h} \psi\right\}\right\|_{\mathcal{F}_{h}^{a}} \lesssim \frac{1}{\sqrt{c_{2}}}\left\|\rho_{a}^{1 / 2} \nabla_{h} \psi\right\|_{\Omega_{a}} & & \forall \psi \in V_{h}^{a}, \\
\left\|\gamma^{-1 / 2}\left\{m \nabla_{h} \cdot \boldsymbol{z}\right\}\right\|_{\mathcal{F}_{h}^{\star}} \lesssim \frac{1}{\sqrt{c_{3}}}\left\|m^{1 / 2} \nabla_{h} \cdot \boldsymbol{z}\right\|_{\Omega_{p}} & & \forall \boldsymbol{z} \in \boldsymbol{V}_{h}^{p},
\end{aligned}
$$

where $c_{1}, c_{2}$ and $c_{3}$ are the constants appearing in (23), (24) and (25), respectively.

The following Lemma establishes the coercivity and boundedness of the discrete bilinear form $\mathcal{A}_{h}$ defined in (21).

Lemma A.2. Let Assumptions 3.1 and 3.2 be satisfied. Then,

$$
\begin{aligned}
& \mathcal{A}_{h}^{e}(\boldsymbol{u}, \boldsymbol{v}) \lesssim\|\boldsymbol{u}\|_{\mathrm{dG}, \mathrm{e}}\|\boldsymbol{v}\|_{\mathrm{dG}, \mathrm{e}} \\
& \mathcal{A}_{h}^{e}(\boldsymbol{u}, \boldsymbol{u}) \gtrsim\|\boldsymbol{u}\|_{\mathrm{dG}, \mathrm{e}}^{2} \\
& \forall \boldsymbol{u}, \boldsymbol{v} \in \boldsymbol{V}_{h}^{p}, \\
& \mathcal{A}_{h}^{p}(\boldsymbol{u}, \boldsymbol{v}) \lesssim|\boldsymbol{u}|_{\mathrm{dG}, \mathrm{p}}|\boldsymbol{v}|_{\mathrm{dG}, \mathrm{p}} \\
& \mathcal{A}_{h}^{p}(\boldsymbol{u}, \boldsymbol{u}) \gtrsim|\boldsymbol{u}|_{\mathrm{dG}, \mathrm{p}}^{2} \\
& \forall \boldsymbol{u}, \boldsymbol{v} \in \boldsymbol{V}_{h}^{p}, \\
& \mathcal{A}_{h}^{a}(\varphi, \psi) \lesssim\|\varphi\|_{\mathrm{dG}, \mathrm{a}}\|\psi\|_{\mathrm{dG}, \mathrm{a}} \\
& \mathcal{A}_{h}^{e}(\boldsymbol{u}, \boldsymbol{v}) \lesssim\|\boldsymbol{u}\|_{\mathrm{dG}, \mathrm{e}}\|\boldsymbol{v}\|_{\mathrm{dG}, \mathrm{e}} \\
& \mathcal{A}_{h}^{a}(\varphi, \varphi) \gtrsim\|\varphi\|_{\mathrm{dG}, \mathrm{a}}^{2} \\
& \forall \varphi, \psi \in V_{h}^{a}, \\
& \mathcal{A}_{h}^{a}(\varphi, \psi) \lesssim\|\varphi\|_{\mathrm{dG}, \mathrm{a}}\|\psi\|_{\mathrm{dG}, \mathrm{a}} \\
& \forall \boldsymbol{u} \in \boldsymbol{H}^{2}\left(\mathcal{T}_{h}^{p}\right) \\
& \forall \boldsymbol{v} \in \boldsymbol{V}_{h}^{p}, \\
& \mathcal{A}_{h}^{p}(\boldsymbol{w}, \boldsymbol{z}) \lesssim\|\boldsymbol{w}\|_{\mathrm{dG}, \mathrm{p}}|\boldsymbol{z}|_{\mathrm{dG}, \mathrm{p}} \\
& \forall \varphi \in H^{2}\left(\mathcal{T}_{h}^{a}\right) \\
& \forall \psi \in V_{h}^{a}, \\
& \forall \boldsymbol{w} \in \boldsymbol{H}^{2}\left(\mathcal{T}_{h}^{p}\right) \\
& \forall \boldsymbol{z} \in \boldsymbol{V}_{h}^{p} \text {. }
\end{aligned}
$$

The coercivity bounds hold provided that the stability parameters $c_{1}, c_{2}$ and $c_{3}$ appearing in (23),(24) and (25), respectively, are chosen sufficiently large.

Proof. The proof is based on employing Lemma A.1 and standard arguments. See also [8] and [4, Lemma A.2]. 


\section{References}

[1] C. Agut and J. Diaz. Stability analysis of the Interior Penalty Discontinuous Galerkin method for the wave equation. ESAIM: Mathematical Modelling and Numerical Analysis Modélisation Mathématique et Analyse Numérique, 47(3):903-932, 2013.

[2] I. Ambartsumyan, E. Khattatov, I. Yotov, and P. Zunino. A Lagrange multiplier method for a Stokes-Biot fluid-poroelastic structure interaction model. Numerische Mathematik, 140(2):513-553, 2018.

[3] P. Antonietti, M. Verani, C. Vergara, and S. Zonca. Numerical solution of fluid-structure interaction problems by means of a high order Discontinuous Galerkin method on polygonal grids. Finite Elem. Anal. Des., 159:1-14, 2019.

[4] P. F. Antonietti, F. Bonaldi, and I. Mazzieri. A high-order discontinuous Galerkin approach to the elasto-acoustic problem. Comput. Methods Appl. Mech. Engrg., 358:112634, 29, 2020.

[5] P. F. Antonietti, F. Bonaldi, and I. Mazzieri. Simulation of three-dimensional elastoacoustic wave propagation based on a Discontinuous Galerkin Spectral Element Method. Internat. J. Numer. Methods Engrg., 121(10):2206-2226, 2020.

[6] P. F. Antonietti, C. Facciolà, A. Russo, and M. Verani. Discontinuous Galerkin Approximation of Flows in Fractured Porous Media on Polytopic Grids. SIAM J. Sci. Comput., 41(1):A109-A138, 2019.

[7] P. F. Antonietti, S. Giani, and P. Houston. hp-version composite Discontinuous Galerkin methods for elliptic problems on complicated domains. SIAM J. Sci. Comput., 35(3):A1417A1439, 2013.

[8] P. F. Antonietti and I. Mazzieri. High-order discontinuous Galerkin methods for the elastodynamics equation on polygonal and polyhedral meshes. Comput. Methods Appl. Mech. Engrg., 342:414-437, 2018.

[9] P. F. Antonietti, I. Mazzieri, M. Muhr, V. Nikolić, and B. Wohlmuth. A high-order discontinuous Galerkin method for nonlinear sound waves. J. Comput phys, 415:109484, 2020.

[10] D. N. Arnold, F. Brezzi, B. Cockburn, and L. D. Marini. Unified analysis of discontinuous Galerkin methods for elliptic problems. SIAM Journal on Numerical Analysis, 39(5):1749$1779,2001 / 02$.

[11] F. Bassi, L. Botti, A. Colombo, D. A. Di Pietro, and P. Tesini. On the flexibility of agglomeration based physical space discontinuous Galerkin discretizations. J. Comput. Phys., 231(1):45-65, 2012.

[12] R. L. Berge, I. Berre, E. Keilegavlen, J. M. Nordbotten, and B. Wohlmuth. Finite volume discretization for poroelastic media with fractures modeled by contact mechanics. International Journal for Numerical Methods in Engineering, 121(4):644-663, 2020.

[13] A. Bermùdez, R. Rodrìguez, and D. Santamarina. Finite element approximation of a displacement formulation for time-domain elastoacoustic vibrations. Journal of Computational and Applied Mathematics, 152(1):17 - 34, 2003.

[14] M. A. Biot. General theory of three-dimensional consolidation. Journal of applied physics, 12(2):155-164, 1941.

[15] L. Botti, M. Botti, and D. A. Di Pietro. An abstract analysis framework for monolithic discretisations of poroelasticity with application to Hybrid High-Order methods. Comput. Math. Appl., June 2020. 
[16] A. Cangiani, Z. Dong, and E. H. Georgoulis. $h p$-version space-time discontinuous Galerkin methods for parabolic problems on prismatic meshes. SIAM J. Sci. Comput., 39(4):A1251A1279, 2017.

[17] A. Cangiani, Z. Dong, and E. H. Georgoulis. $h p$-version discontinuous Galerkin methods on essentially arbitrarily-shaped elements, 2020.

[18] A. Cangiani, Z. Dong, E. H. Georgoulis, and P. Houston. hp-version discontinuous Galerkin methods for advection-diffusion-reaction problems on polytopic meshes. ESAIM Math. Model. Numer. Anal., 50(3):699-725, 2016.

[19] A. Cangiani, Z. Dong, E. H. Georgoulis, and P. Houston. hp-version discontinuous Galerkin methods on polytopic meshes. SpringerBriefs in Mathematics. Springer International Publishing, 2017.

[20] A. Cangiani, E. H. Georgoulis, and P. Houston. hp-version discontinuous Galerkin methods on polygonal and polyhedral meshes. Mathematical Models and Methods in Applied Sciences, 24(10):2009-2041, 2014.

[21] J. Carcione. Wave Fields in Real Media, volume 38. Elsevier Science, 2014.

[22] B. Castagnede, A. Aknine, M. Melon, and C. Depollier. Ultrasonic characterization of the anisotropic behavior of air-saturated porous materials. Ultrasonics, 36(1-5):323-341, 1998.

[23] G. Chiavassa and B. Lombard. Wave propagation across acoustic/Biot's media: A finitedifference method. Communications in Computational Physics, 13(4):985-1012, 2013.

[24] S. Congreve and P. Houston. Two-grid hp-DGFEMs on agglomerated coarse meshes. PAMM, 19(1):e201900175, 2019.

[25] J. de la Puente, M. Dumbser, M. Käser, and H. Igel. Discontinuous Galerkin methods for wave propagation in poroelastic media. Geophysics, 73(5):T77-T97, 2008.

[26] M. Dryja and M. Sarkis. Additive average schwarz methods for discretization of elliptic problems with highly discontinuous coefficients. Computational Methods in Applied Mathematics, 10(2):164 - 176, 2010.

[27] A. Ezziani. Modélisation mathématique et numérique de la propagation d'ondes dans les milieux viscoélastiques et poroélastiques. Theses, ENSTA ParisTech, 2005.

[28] B. Flemisch, M. Kaltenbacher, S. Triebenbacher, and B. Wohlmuth. The equivalence of standard and mixed finite element methods in applications to elasto-acoustic interaction. SIAM J. Sci. Comput., 32(4):1980-2006, 2010.

[29] B. Flemisch, M. Kaltenbacher, and B. Wohlmuth. Elasto-acoustic and acoustic-acoustic coupling on non-matching grids. Internat. J. Numer. Methods Engrg., 67:1791-1810, 2006.

[30] M. Grote, A. Schneebeli, and D. Schötzau. Discontinuous Galerkin finite element method for the wave equation. SIAM J. Numer. Anal., 44(6):2408-2431, 2006.

[31] B. Gurevich and M. Schoenberg. Interface conditions for Biot's equations of poroelasticity. J. Acoust. Soc. Am., 105(5):2585-2589, 1999.

[32] T. Haire and C. Langton. Biot theory: a review of its application to ultrasound propagation through cancellous bone. Bone, 24(4):291 - 295, 1999.

[33] J. M. Huyghe, D. H. van Campen, T. Arts, and R. M. Heethaar. A two-phase finite element model of the diastolic left ventricle. Journal of biomechanics, 24(7):527-538, 1991. 
[34] G. Jayaraman. Water transport in the arterial wall-a theoretical study. Journal of biomechanics, 16(10):833-840, 1983.

[35] B. Krishnan, D. M., S. Raja, and K. Venkataramana. Structural and Vibroacoustic Analysis of Aircraft Fuselage Section with Passive Noise Reducing Materials: A Material Performance Study. 032015.

[36] B. Lombard and J. Piraux. Numerical treatment of two-dimensional interfaces for acoustic and elastic waves. Journal of Computational Physics, 195(1):90 - 116, 2004.

[37] P. J. Matuszyk and L. F. Demkowicz. Solution of coupled poroelastic/acoustic/elastic wave propagation problems using automatic hp-adaptivity. Comput. Methods Appl. Mech. Engrg., 281:54-80, 2014.

[38] C. Morency and J. Tromp. Spectral-element simulations of wave propagation in porous media. Geophysical Journal International, 175(1):301-345, 2008.

[39] C. Oomens, D. Van Campen, and H. Grootenboer. A mixture approach to the mechanics of skin. Journal of biomechanics, 20(9):877-885, 1987.

[40] I. Perugia and D. Schötzau. An $h p$-analysis of the local discontinuous Galerkin method for diffusion problems. J. Sci. Comput., 17(1):561-571, 2002.

[41] P. J. Phillips and M. F. Wheeler. A coupling of mixed and discontinuous Galerkin finiteelement methods for poroelasticity. Computational Geosciences, 12(4):417-435, 2008.

[42] A. Quarteroni. Numerical models for differential problems, volume 8. Springer-Verlag Mailand, 2014.

[43] B. Rivière and M. F. Wheeler. Discontinuous finite element methods for acoustic and elastic wave problems. Contemporary Mathematics, 329:271-282, 2003.

[44] R. T. Rockafellar. Lagrange multipliers and optimality. SIAM review, 35(2):183-238, 1993.

[45] R. Sidler, J. M. Carcione, and K. Holliger. Simulation of surface waves in porous media. Geophysical Journal International, 183(2):820-832, 2010.

[46] M. Souzanchi, L. Cardoso, and S. Cowin. Tortuosity and the averaging of microvelocity fields in poroelasticity. Journal of applied mechanics, 80(2), 2013.

[47] E. M. Stein. Singular integrals and differentiability properties of functions, volume 2. Princeton University Press, 1970.

[48] C. Talischi, G. H. Paulino, A. Pereira, and I. F. Menezes. Polymesher: a general-purpose mesh generator for polygonal elements written in Matlab. Structural and Multidisciplinary Optimization, 45(3):309-328, 2012.

[49] S. Triebenbacher, M. Kaltenbacher, B. Wohlmuth, and B. Flemisch. Applications of the mortar finite element method in vibroacoustics and flow induced noise computations. Acta Acustica united with Acustica, 96(3):536-553(18), 2010. 